\title{
TIME DECAY ESTIMATES FOR WAVE EQUATIONS WITH TRANSMISSION AND BOUNDARY CONDITIONS
}

\author{
KREŠO MiHALINČIĆ \\ University of Rijeka, Croatia
}

\begin{abstract}
Time decay estimates are derived for solutions of some initial value problems of wave propagation, based on the method of stationary phase. Solutions to three dimensional wave equation in wedges and one dimensional wave equation with a constant potential are shown to decay like $t^{-1}$ and $t^{-1 / 2}$, respectively. Dependencies of the results on initial data and physical implications are discussed.
\end{abstract}

\section{INTRODUCTION}

In this paper we consider the central problem given in Figure 1 (a rigorous function analytical formulation was developed in [6]; we present it in section 2). Here,

$$
K_{1 /(2)}=\{X:=(x, y, z): x<0(x>0), y>0, z \in \mathbb{R}\}
$$

denote two adjacent rectangular wedges in the upper half space $(y>0)$ of $\mathbb{R}^{3}$ also denoted by $\mathbb{R}_{+}^{3}, a_{1}, a_{2}$ are real positive constants with $a_{1}<a_{2}$ and we look for such functions $f(t, x, y, z)$ that satisfy the linear wave equation

$$
\partial_{t}^{2} f(t, X)-a(x) \Delta f(t, X)=0, \Delta=\partial_{x}^{2}+\partial_{y}^{2}+\partial_{z}^{2}
$$

in $K_{1}, K_{2}$ with $a(x)=a_{1}$ for $x<0, a(x)=a_{2}$ for $x>0$ and initial conditions

$$
u_{0}(X)=f(t=0, X), \quad u_{1}(X)=\frac{\partial f}{\partial t}(t=0, X),
$$

Dirichlet boundary condition on the bottom of the wedges

$$
f(x, 0, z)=0
$$

2010 Mathematics Subject Classification. 35L05, 74S25, 35G16.

Key words and phrases. Partial differential equations, initial boundary value problem, mathematical physics, wave propagation, stationary phase, time decay. 


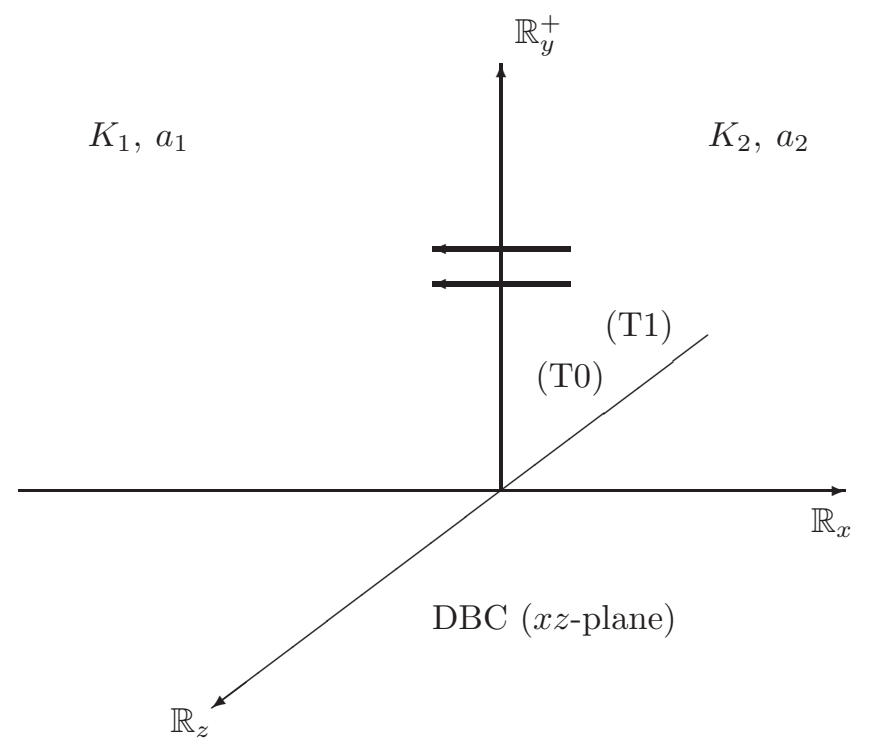

Figure 1. Two material wedges with transmission and Dirichlet conditions

and transmission conditions on their interface $\left(d_{1}, d_{2}\right.$ are positive constants)

$$
\begin{aligned}
f\left(0^{-}, y, z\right) & =f\left(0^{+}, y, z\right), \\
d_{1} \frac{\partial f}{\partial x}\left(0^{-}, y, z\right) & =d_{2} \frac{\partial f}{\partial x}\left(0^{+}, y, z\right) .
\end{aligned}
$$

Solutions to this and two related problems were derived in our previous articles: wave equation in three dimensional half space (a special case of our present problem without transmission i.e. $a_{1}=a_{2}$ ) was solved in [23] while wave equation in one space dimension with a constant potential was solved in a natural coupling with our three dimensional problem in $[6] .^{1}$

Now, once the solutions have been provided, the question of their time asymptotic behavior arises. The asymptotics of the half space problem were discussed in [23]. In the present paper $^{2}$ we turn to the latter two problems and utilize their close connection demonstrated in [6] to derive respective time decay estimates simultaneously. Generally, research of this kind contributes to

\footnotetext{
${ }^{1}$ In that context, it was called Klein-Gordon equation in one dimension with potential step to keep in line with [4].

${ }^{2}$ This paper is a revised version of the author's Ph.D. thesis, cf. [22] mentored by F. Ali Mehmeti, H.-D. Alber and E. Meister at the Technical University of Darmstadt, Germany. I am especially indebted to O. Liess for his guidance.
} 
the understanding of wave phenomena on complicated geometric structures. For a comprehensive outline of the underlying concepts we refer to Meister, [21]. Our primary concern is the linearized equation of sound wave propagation given by (1.2). As a rule, one first seeks some information on time decay of this equation. Such information, interesting in itself, may then serve as a basis for investigation of related nonlinear problems like those of existence and uniqueness (e.g. [24]).

If no constraints other than the initial conditions are imposed on (1.2), one talks of a full space problem ${ }^{3}$. It has been studied extensively; we only refer to classic papers by John ([15]), John and Klainerman ([16]), Klainerman and Ponce ([17]) and v. Wahl ([26]). They show that global solutions (for all times) do not exist in general, leading to the question of life span of the solution. Analyses of the pertaining linear equation are based on the closed solution $\left(a_{1}=a_{2}=a\right.$ and $\Omega_{3}$ is the unit sphere in $\left.\mathbb{R}^{3}\right)$

$$
u_{F}(t, X)=\frac{t}{4 \pi \sqrt{a}} \int_{\Omega_{3}} u_{1}(X+\sqrt{a} t \xi) d \xi
$$

from which a time decay result of $L^{1}-L^{\infty}$-type

$$
\left\|u_{F}\right\|_{L^{\infty}} \leq \text { const. } t^{-1}\left\|u_{1}\right\|_{W^{1,1}}
$$

follows in an elementary way (cf. [26]).

Now, in order to simulate wave propagation in presence of obstacles causing reflection, scattering and diffraction effects, one prescribes certain boundary conditions in addition to the initial values. A variety of perturbations to the full space problem have been considered in literature. Zachmanoglou (cf. [29]) showed that the solution of the wave equation in exterior domains of certain bodies (including those with infinite boundaries) decays like $t^{-1}$ at any fixed point and like $t^{-2}$ within finite spheres. Beals and Strauss establish criteria on potentials that leave decay rates of wave equations unchanged $([9])$. Alber and Leis (cf. [2], [18]) discuss a variety of exterior problems including large time and high frequency asymptotics. Resonance phenomena arising from a combination of a periodic spatial structure and a time-periodic force are observed by Werner in [27].

More recently, wave equations with potentials and/or damping have been studied by a number of authors. In [12] faster than $t^{-\frac{1}{2}}$ decay rates for wave equation with a decaying potential are derived. In [10] polynomial decay rates for linear damping in exterior domains are derived. For nonlinear damping of

\footnotetext{
${ }^{3}$ Usually, initially unperturbed media i.e. $u_{0} \equiv 0$ is observed and only initial disturbance $u_{1}$ prescribed, see solution to full space problem few lines below. As [6] solves for $u_{0} \neq 0$, we quote the solution in section 2 , setting $u_{0} \equiv 0$ from section 3 on. Still, all our procedures apply to the solution with $u_{0} \neq 0$, as will become clear throughout the paper.
} 
Klein-Gordon equation in [7] exponential decay rates in the exterior of a starshaped obstacle are derived, while in [11] subtler estimates for wave equation are proven.

Literature on transmission problems is comparatively scarce. In [8] and [14] it is shown that solutions to wave equation in bodies consisting of two different materials decay at the same (polynomial or exponential) rates as corresponding relaxation functions. In [5] $t^{-\frac{1}{2}}$ decay rates are derived for the Klein-Gordon equation on a star-shaped network building up on [22].

Of crucial importance from the standpoint of interpretation is whether time decay rates in presence of perturbations change with respect to the unperturbed situation. As shown in [20], $L^{\infty}$-decay for the free Klein-Gordon equation is given by $t^{-\frac{n}{2}}$ in $n$ space dimensions (as opposed to $t^{-\frac{n-1}{2}}$ for wave equation). While [20] utilizes van der Corput Lemma, Liess in [19] uses the method of stationary phase (cf. [13]) also adopted in our paper. The complicated structure of the system of crystal optics caused a loss of the decay rate with respect to the full space problem, as reflected in an estimate by $t^{-\frac{1}{2}}$. We will recover the "full" estimate by $t^{-1}$ for wave equation with transmission due to a feature inherent to the problem in section 5 .

Our paper is organized as follows: in section 2, we recall our formulation from $[6]$ in terms of a selfadjoint operator A and quote the solution obtained there. For the purpose of this formulation, we introduced sesquilinear forms (for sufficiently smooth $f_{j}, g_{j}$ and index $j$ denoting restrictions of $f, g$ to $K_{j}$, $j=1,2$, see section 2 for details)

$$
b_{j}\left(f_{j}, g_{j}\right)=\int_{K_{j}}\left(\Delta f_{j}\right) \bar{g}_{j} d X+\int_{K_{j}} \nabla f_{j} \nabla \bar{g}_{j} d X, j=1,2,
$$

and modified our transmission condition (T1) as

$$
d_{1} b_{1}\left(f_{1}, g_{1}\right)+d_{2} b_{2}\left(f_{2}, g_{2}\right)=0, \quad \forall g_{j} \in H_{1}\left(K_{j}\right) .
$$

Actual work of this paper starts in section 3 where a number of transformations are undertaken to express this solution in terms of oscillating integrals. In order to derive a time decay result of an $L^{1}-L^{\infty}$ - type we apply the method of stationary phase from [13] to the oscillating integrals that make up the solution. In doing so, we keep in mind parameter dependencies of integrals in question and their phases. Ensuing technical difficulties lead to some restrictions of initial data in sections 4 through 6 , in which we also derive decay rates for wave equation in one space dimension with a constant potential. Nevertheless, for wave equation we arrive at an estimate with $t^{-1}$ :

$$
\|u\|_{\infty} \leq \text { const. } t^{-1}\left\|u_{1}\right\|_{L^{1,2}\left(\mathbb{R}_{+}^{3} ; X\right)}
$$

and for wave equation in one space dimension with a constant potential at a similar estimate with $t^{-1 / 2}$. In the final section we extend these results to 
the physically interesting "test elements" i.e. initial data from $\mathcal{C}_{0}^{\infty}$ (smooth functions with compact support).

In conclusion, we highlight some physical implications of our results. First, our solutions are expressed in terms of oscillating integrals (section 3 ) that involve a variety of integral transforms of initial data: complex in $z \in(-\infty, \infty)$ direction mapping $z \mapsto \zeta$, sine in $y>0$ direction $(y \mapsto \eta)$ and generalized via generalized eigenfunctions in the perturbed $x$-direction $(x \mapsto$ $\mu \mapsto q)$. Eventually, these transforms produce - with $p=(\eta, \zeta), P=(p, q)$ phases of type

$$
f_{k}^{j}\left(p ; q_{j}, S\right)=\sqrt{a\left(|p|^{2}+q^{2}\right)} t-\eta y-\zeta z-q x .
$$

For a fixed $q$, the above formula reduces to the phase of the integral solution to the two dimensional Klein-Gordon equation whose phase reads $f_{K G}=$ $\sqrt{a|p|^{2}+m} t-\eta y-\zeta z, m=a q^{2} \in \mathbb{R}$. Indeed, in section 5 , stationary phase considerations forced us to keep this third integration variable $q$ fixed which resulted in a reduction of the decay rate by one $\left(2 \frac{-1}{2}\right.$ rather than $3 \frac{-1}{2}$ for a three dimensional Klein-Gordon equation). This observation is immediately extended to any space dimension $n$ showing that time decay rates for the wave and the Klein-Gordon equation are given by $t^{-\frac{n-1}{2}}$ and $t^{-\frac{n}{2}}$, respectively.

Second, the closed form of the solution to the full- and half- space problems readily implies that sharp signals (described by Dirac's $\delta$ functional, e.g. [30]) sent from the origin at a time $t=0$ propagate in sharp fronts. This means that if we set $u_{1}(X)=\delta_{0}(X)$ then an observer at the point $X$ receives the signal at the time $t=\frac{1}{\sqrt{a}}|X|$ and has silence for all other times; one also says that Huygens' principle holds. In the presence of transmission effects we show that an observer at the point $X \in K_{1}\left(X \in K_{2}\right)$ only receives the signal at the time

$$
t_{1}=\frac{1}{\sqrt{a_{1}}}|X| \quad\left(t_{2}=\frac{1}{\sqrt{a_{2}}}|X|\right),
$$

respectively. This statement is now of asymptotic nature (sections 4,5 ). Essentially, sound spreads in two decoupled media with velocities $\sqrt{a_{1}}$ in $K_{1}$ and $\sqrt{a_{2}}$ in $K_{2}$ in line with the interaction model of Ali Mehmeti (cf. [3]). Whether this model remains valid in nonlinear situations is an interesting question for future investigation.

\section{Function Analytical Framework}

We defined the two wedges $K_{1}, K_{2}$ in the introduction under (1.1). We also defined a Dirichlet boundary condition denoted by (DBC) and two transmission conditions (T0), (T1'). We now provide the relevant highlights from [6]. The fundamental Hilbert space is the product space

$$
\mathcal{H}=L_{2}\left(\mathbb{R}_{3}^{+}\right)=\prod_{j=1}^{2} L_{2}\left(K_{j}\right) .
$$


For sufficiently regular functions $f$ we write

$$
\frac{\partial}{\partial x} f=\partial_{1} f=\left(\partial_{1} f_{1}, \partial_{1} f_{2}\right)
$$

and analogously for partial derivatives with respect to $y$ and $z$ variables

$$
\frac{\partial}{\partial y} f=\partial_{2} f, \quad \frac{\partial}{\partial z} f=\partial_{3} f
$$

We introduce Standard Sobolev spaces as

$$
W^{l, m}\left(K_{j}\right)=\left\{f_{j} \in L_{l}\left(K_{j}\right): D^{\alpha} f_{j} \in L_{l}\left(K_{j}\right):|\alpha| \leq m\right\}
$$

where $\alpha$ denotes the multiindex. In particular, $H^{m}\left(K_{j}\right)=W^{2, m}\left(K_{j}\right)$ and, with $\Delta=\partial_{x}^{2}+\partial_{y}^{2}+\partial_{z}^{2}$ denoting the (weak) Laplace-Operator,

$$
H^{1}\left(\Delta, K_{j}\right):=\left\{f_{j} \in H^{1}\left(K_{j}\right): \Delta f_{j} \in L_{2}\left(K_{j}\right)\right\} .
$$

The space of infinitely many times differentiable functions with compact support is denoted by $\mathcal{C}_{0}^{\infty}\left(K_{j}\right)$. For the more detailed information on the spaces introduced here we refer to [1]. Of particular importance for our estimates are weighted $L_{1}$ spaces with the norm (for any $m \in N$ )

$$
\|f\|_{L^{1, m}\left(\mathbb{R}_{+}^{3} ; X\right)}=\sum_{|\alpha| \leq m_{\mathbb{R}_{+}^{3}}} \int_{X^{\alpha} f(X) \mid d X .}
$$

In sections 4 through 6 we will endow some function spaces of interest with this norm and derive corresponding estimates.

Definition 2.1. The operator $(A, D(A))$ - A for short - is defined by the fundamental Hilbert space $\mathcal{H}$, its domain is given by

$$
D(A)=\prod_{j=1}^{2} H^{1}\left(\Delta, K_{j}\right) \cap\{f \in \mathcal{H}: f \text { satisfies (DBC), (T0), (T1') }\}
$$

and its action is given by

$$
\text { Af }=\left(A_{1} f_{1}, A_{2} f_{2}\right) \quad \text { with } \quad A_{j} f_{j}=-a_{j} \Delta f_{j} .
$$

We next considered the formal eigenvalue equation (for $u \in D(A)$ as in Definition 2.1)

$$
A_{j} f_{j}(x, y, z)=\mu f_{j}(x, y, z), \quad(x, y, z) \in K_{j}, \quad j=1,2 .
$$

Observing that an application of Fourier sine transformation in $y$-direction $(y \mapsto \eta)$ and complex Fourier transformation in $z$-direction $(z \mapsto \zeta)$ leads to

$$
-a_{j} \partial_{x}^{2}\left(\mathcal{F} f_{j}\right)(x, \eta, \zeta)+a_{j}|p|^{2}\left(\mathcal{F} f_{j}\right)(x, \eta, \zeta)=\mu \mathcal{F} f_{j}(x, \eta, \zeta)
$$

we introduced another operator $A_{p} f_{j}:=-a_{j} \partial_{x}^{2} f_{j}+a_{j}|p|^{2} f_{j}$ and noted that, for a fixed parameter $p=(\eta, \zeta)$, our problem reduces to one space dimension problem with formal eigenvalue equation

$$
A_{p} f_{j}:=-a_{j} \partial_{x}^{2} f_{j}+c_{j} f_{j}=\mu f_{j}, c_{j}=a_{j}|p|^{2} .
$$


As this turned out to be the eigenvalue equation for Klein-Gordon equation with transmission from [4], we revisited this operator, called it $A^{1}-$ or $A_{p}$ to denote the observed parameter dependence of $c_{j}$ - and used it to discuss our original operator $A$.

Definition 2.2. Let $N_{1}=[-\infty, 0)$ and $N_{2}=[0, \infty)$ denote the two half axes of $\mathbb{R}$ and let the transmission conditions be given by

$$
\begin{aligned}
f_{1}\left(0^{-}\right) & =f_{2}\left(0^{+}\right), \\
d_{1} \partial_{x} f_{1}\left(0^{-}\right) & =d_{2} \partial_{x} f_{2}\left(0^{+}\right) .
\end{aligned}
$$

We define the fundamental real Hilbert space

$$
\mathcal{H}^{1}=\prod_{j=1}^{2} L_{2}\left(N_{j}\right)
$$

and the operator $\left(A^{1}, D\left(A^{1}\right)\right)-A^{1}$ for short - by its domain

$$
D\left(A^{1}\right)=\prod_{j=1}^{2} H^{2}\left(N_{j}\right) \cap\left\{f_{j} \in \mathcal{H}^{1}: f_{j} \text { satisfies }\left(T 0_{1}\right) \text { and }\left(T 1_{1}\right)\right\}
$$

and its action

$$
A_{j}^{1} f_{j}=-a_{j} \partial_{x}^{2} f_{j}+c_{j} f_{j}, \quad j=1,2, \quad c_{2}>c_{1}>0 .
$$

Having shown self-adjointness of $A$ and $A^{1}$, we were able to restate and solve corresponding initial boundary value problems within our function analytical framework as follows:

Definition 2.3 (Problem statement for three dimensional wave equation in wedges). Find functions $u(t ; x, y, z)$ satisfying

$$
\begin{aligned}
& \partial_{t}^{2} u+A u=0, \\
& u_{0}(x, y, z)=u(t=0 ; x, y, z), \\
& u_{1}(x, y, z)=\partial_{t} u(t=0 ; x, y, z),
\end{aligned}
$$

with initial conditions $u_{0}, u_{1} \in D\left(A^{\frac{1}{2}}\right)=V$ where (cf. [6])

$$
V=\prod_{j=1}^{2} H_{1}\left(K_{j}\right) \cap\{u \in \mathcal{H}: u \text { satisfies }(D B C),(T 0)\} .
$$

We define the initial boundary value problem for $A^{1}$ in similar fashion.

Definition 2.4 (Problem statement for wave equation in one space dimension with a constant potential). Find functions $u(t ; x, y, z)$ satisfying

$$
\begin{aligned}
& \partial_{t}^{2} u+A^{1} u=0, \\
& u_{0}(x)=u(t=0 ; x), \\
& u_{1}(x)=\partial_{t} u(t=0 ; x),
\end{aligned}
$$


with initial conditions $u_{0}, u_{1} \in D\left(\left(A^{1}\right)^{\frac{1}{2}}\right)=V^{1}$ where (cf. [6])

$$
V^{1}=\prod_{j=1}^{2} H^{1}\left(N_{j}\right) \cap\left\{f_{j} \in \mathcal{H}^{1}: f_{j} \text { satisfies }\left(T 0_{1}\right)\right\} .
$$

We obtain the following expressions for $A^{1}$ and $A$.

THEOREM 2.5 (Solution of wave equation in one space dimension with a constant potential). Initial boundary value problem of Definition 2.4 with $c_{1}=a_{1}|p|^{2}, c_{2}=a_{2}|p|^{2}$ for a fixed $p$ and $A^{1}$ understood as $A_{p}$ has the solution

$$
\begin{aligned}
u(t, x ; p)= & \frac{1}{\pi d_{1}} \sum_{k=0}^{2} \int_{J_{k}^{1}} \cos (\sqrt{\alpha} t)\left(u_{0}\right)_{k}(\alpha ; p) \bar{E}_{k}(x ; p, \alpha) d \alpha \\
& +\frac{1}{\pi d_{1}} \sum_{k=0}^{2} \int_{J_{k}^{1}} \frac{\sin (\sqrt{\alpha} t)}{\sqrt{\alpha}}\left(u_{1}\right) \tilde{k}(\alpha ; p) \bar{E}_{k}(x ; p, \alpha) d \alpha
\end{aligned}
$$

where

$$
J_{k}^{1}=J_{k}^{1}(p):=\left\{\begin{array}{c}
\left(a_{1}|p|^{2}, a_{2}|p|^{2}\right), k=0 \\
\left(a_{2}|p|^{2}, \infty\right), k=1,2
\end{array} \quad\left(\text { i.e. } c_{j}=a_{j}|p|^{2}\right),\right.
$$

$$
E_{0}(x ; p, \alpha)=\left\{\begin{array}{c}
\beta_{0}(\alpha)\left[\cos \left(\tilde{\xi}_{1}(\alpha) x\right)-\frac{d_{2} \tilde{\xi}_{2}(\alpha)}{d_{1} \tilde{\xi}_{1}(\alpha)} \sin \left(\tilde{\xi}_{1}(\alpha) x\right)\right]=\left(E_{0}(x ; p, \alpha)\right)_{1} \\
\beta_{0}(\alpha) \exp \left(-\xi_{2}(\alpha) x\right)=\left(E_{0}(x ; p, \alpha)\right)_{2}
\end{array},\right.
$$

for $0<c_{1}=a_{1}|p|^{2}<\alpha<c_{2}=a_{2}|p|^{2} \quad$ and

$$
\begin{aligned}
& E_{1}(x ; p, \alpha)=\left\{\begin{array}{l}
\beta_{1}(\alpha) \cos \left(\tilde{\xi}_{1}(\alpha) x\right)=\left(E_{1}(x ; p, \alpha)\right)_{1} \\
\beta_{1}(\alpha) \cos \left(\tilde{\xi_{2}}(\alpha) x\right)=\left(E_{1}(x ; p, \alpha)\right)_{2}
\end{array},\right. \\
& E_{2}(x ; p, \alpha)=\left\{\begin{array}{l}
\frac{\beta_{2}(\alpha)}{d_{1} \tilde{\xi}_{1}(\alpha)} \sin \left(\tilde{\xi_{1}}(\alpha) x\right)=\left(E_{2}(x ; p, \alpha)\right)_{1} \\
\frac{\beta_{2}(\alpha)}{d_{2} \xi_{2}(\alpha)} \sin \left(\tilde{\xi_{2}}(\alpha) x\right)=\left(E_{2}(x ; p, \alpha)\right)_{2}
\end{array}\right.
\end{aligned}
$$

for $0<c_{1}=a_{1}|p|^{2}<c_{2}=a_{2}|p|^{2}<\alpha$ are generalized eigenfunctions of $A^{1}$ i.e. $A_{p}$. Further, $\tilde{\xi}_{j}=\tilde{\xi}_{j}(p, \alpha)$ and $\beta_{k}(\alpha)$ are given by

$$
\tilde{\xi}_{j}(\alpha)=\tilde{\xi}_{j}(p, \alpha)= \begin{cases}\sqrt{\left(\alpha-c_{j}\right) / a_{j}}, & \alpha>c_{j}=a_{j}|p|^{2} \\ \sqrt{\left(c_{j}-\alpha\right) / a_{j}}, & \alpha<c_{j}=a_{j}|p|^{2}\end{cases}
$$

and

$$
\beta_{0}(\alpha)^{2}=\frac{d_{1}^{2} \tilde{\xi}_{1}}{d_{1}^{2} \tilde{\xi}_{1}^{2}+d_{2}^{2} \tilde{\xi}_{2}^{2}}, \beta_{1}(\alpha)^{2}=\frac{d_{1}}{d_{1} \tilde{\xi}_{1}+d_{2} \tilde{\xi}_{2}}, \beta_{2}(\alpha)^{2}=\frac{d_{1}^{2} d_{2} \tilde{\xi_{1}} \tilde{\xi}_{2}}{d_{1} \tilde{\xi}_{1}+d_{2} \tilde{\xi}_{2}} .
$$

Finally, integral transforms $\left(u_{0}\right)_{\tilde{k}},\left(u_{1}\right)_{\tilde{k}}$ are understood as

$$
\tilde{f}_{k}(\alpha ; p):=\sum_{j=1}^{2} \int_{N_{j}}\left(E_{k}(x ; p, \alpha)\right)_{j} f_{j}(x ; p) \frac{d_{j}}{a_{j}} d x, k=0,1,2 .
$$


Obviously, for $\left.E_{k}(x ; p, \alpha)\right)_{j}$ as above, functions defined as

$$
\left(E_{k}(x, y, z ; p, \alpha)\right)_{j}:=\frac{1}{\pi} \sin (\eta y) e^{i \zeta z}\left(E_{k}(x ; p, \alpha)\right)_{j}
$$

solve formal eigenvalue equation for $A$. In fact, with

$$
\tilde{f}_{k}(p, \alpha):=\sum_{j=1}^{2} \int_{K_{j}} f_{j}(x, y, z)\left(E_{k}(x, y, z ; p, \alpha)\right)_{j} \frac{d_{j}}{a_{j}} d X, f \in \mathcal{H}, k=0,1,2,
$$

we were able to show the following result.

THEOREM 2.6 (Solution of 3D wave equation in wedges). Initial boundary value problem of Definition 2.3 has the solution

$$
\begin{aligned}
& u(t ; x, y, z)= \\
& =\frac{1}{\pi d_{1}} \int_{0}^{\infty} \cos (\sqrt{\alpha} t) \sum_{k=0}^{2} \int_{J_{k}}\left(u_{0}\right)_{k}^{\sim}(p, \alpha) \bar{E}_{k}(x, y, z ; p, \alpha) d p d \alpha \\
& \quad+\frac{1}{\pi d_{1}} \int_{0}^{\infty} \frac{\sin (\sqrt{\alpha} t)}{\sqrt{\alpha}} \sum_{k=0}^{2} \int_{J_{k}}\left(u_{1}\right)_{k}^{\sim}(p, \alpha) \bar{E}_{k}(x, y, z ; p, \alpha) d p d \alpha
\end{aligned}
$$

where

$$
J_{k}=\left\{\begin{array}{l}
\left\{p=(\eta, \zeta): 0 \leq|p|^{2} \leq \frac{\alpha}{a_{2}}\right\}, k=1,2 \\
\left\{p=(\eta, \zeta): \frac{\alpha}{a_{2}} \leq|p|^{2} \leq \frac{\alpha}{a_{1}}\right\}, k=0
\end{array}\right.
$$

With this brief review of [6], we can start our analysis of time asymptotics of the above solutions.

\section{Modifications of the Solution}

From now on we set $u_{0} \equiv 0$. In order to estimate our solution via stationary phase method, we introduce the concept of an oscillating integral and modify our integral solution accordingly.

Definition 3.1. Any integral over some subspace of $\mathbb{R}^{n}$ with $\lambda>0$ and $\nu$ in some set of parameters of type

$$
I(\lambda ; \nu)=\int u(\theta, \nu) \exp [i(\lambda f(\theta, \nu))] d \theta
$$

will be called an oscillating integral with phase $f$ and amplitude $u$. As integrals of this kind are usually considered for $\lambda \rightarrow \infty$ we sometimes refer to $\lambda$ as a large parameter.

The series of rather elementary lemmas that follow transform our solution into a form that corresponds to the above definition and proves convenient for estimates via stationary phase. We also set $\xi_{j}:=\tilde{\xi}_{j}, j=1,2$ (this distinction played a role in [6] and we kept it in previous section to keep in line with that paper). 
LEMma 3.2. The solution from Theorem 2.6 can be written as

$$
u(t, X)=\sum_{(k, j), \pm} \alpha_{k}^{j, \pm} I_{k}^{j, \pm}(t, X),(k, j) \in\{(0,1,2)\} \times\{(1,2)\}
$$

where $\alpha_{k}^{j, \pm}$ are some complex constants depending only on $a_{1}, a_{2}, d_{1}, d_{2}$. For $(k, j) \neq(0,2)$ it holds

$$
I_{k}^{j, \pm}(t, X)=\int_{\Omega_{k}} \frac{B_{k}^{j}(|p|, \mu)}{\sqrt{\mu}}\left(u_{1}\right)_{k}^{\sim}(p, \mu) \exp \left[i\left( \pm \sqrt{\mu} t-p x_{2} \mp \xi_{j} x\right)\right] d p d \mu
$$

and for $(k, j)=(0,2)$

$$
I_{0}^{2, \pm}(t, X)=\int_{\Omega_{0}} \frac{B_{0}^{2}(|p|, \mu)}{\sqrt{\mu}}\left(u_{1}\right)_{0}^{\sim}(p, \mu) e^{-\xi_{2} x} \exp \left[i\left( \pm \sqrt{\mu} t-p x_{2}\right)\right] d p d \mu .
$$

Here $p:=\left(p_{1}, p_{2}\right)=(\eta, \zeta)$ and $x_{2}=(y, z)$ and (with some constants $b_{k}^{j}=$ $\left.b_{k}^{j}\left(d_{1}, d_{2}\right)\right)$

$$
\begin{array}{r}
B_{0}^{1}=\beta_{0}(|p|, \mu)+b_{0}^{1} \frac{\xi_{2}}{\sqrt{\xi_{1}}} \beta_{0}(|p|, \mu), b_{0}^{1} \in \mathbf{C}, \\
B_{0}^{2}=b_{0}^{2} \beta_{0}(|p|, \mu), B_{1}^{j}=b_{1}^{j} \beta_{1}(|p|, \mu), B_{2}^{j}=b_{2}^{j} \frac{\beta_{2}(|p|, \mu)}{\xi_{j}} .
\end{array}
$$

This is an immediate consequence of Theorems 2.5, 2.6. Recalling that (for any real $a, \cos a=\frac{1}{2}\left(\mathrm{e}^{i a}+\mathrm{e}^{-i a}\right), \sin a=\frac{1}{2 i}\left(\mathrm{e}^{i a}-\mathrm{e}^{-i a}\right)$, we rewrote the sine and cosine terms as complex functions and the integration areas $J_{k}$ as paraboloids

$$
\Omega_{0}=\left\{(p, \mu): a_{1}|p|^{2}<\mu<a_{2}|p|^{2}\right\}, k=0
$$

and

$$
\Omega_{1}=\Omega_{2}=\left\{(p, \mu): a_{2}|p|^{2}<\mu\right\}, k=1,2 .
$$

We do not give the constants involved in detail as they play no role in subsequent asymptotic analysis of $I_{k}^{j, \pm}(t, X)$; just note that for $X \in K_{1}\left(X \in K_{2}\right)$ it holds $\alpha_{k}^{2, \pm}=0\left(\alpha_{k}^{1, \pm}=0\right) \forall k=0,1,2$. For the same reason we omit \pm -notation for different choices of signs in the phases $I_{k}^{j, \pm}(t, X)$ unless a particular choice actually matters. This will be the case when we prove optimality of our estimates.

Next transformation is in line with common treatment of full-space problems via Fourier analysis (cf. [13], [28] for example):

$$
(p, \mu) \longrightarrow\left(p, a_{j}\left(|p|^{2}+q_{j}^{2}\right)\right), q_{j}:=\sqrt{\frac{\mu-a_{j}|p|^{2}}{a_{j}}}, j=1,2
$$


for the paraboloid $\Omega_{1}=\Omega_{2}$ and for $\Omega_{0}$

$$
(p, \mu) \longrightarrow\left(p, a_{2}\left(|p|^{2}-q^{2}\right)\right), q:=-\sqrt{\frac{a_{2}|p|^{2}-\mu}{a_{2}}}
$$

with new coordinates $P_{j}=\left(p, q_{j}\right)$ and $P=(p, q)$, respectively. Due to

$$
\begin{aligned}
& \operatorname{det}\left(\frac{\partial(p, \mu)}{\partial P_{j}}\right)=2 a_{j} q_{j}, \quad(k, j) \neq(0,2) ; \\
& \operatorname{det}\left(\frac{\partial(p, \mu)}{\partial P}\right)=-2 a_{2} q, \quad(k, j)=(0,2)
\end{aligned}
$$

our transformations are local diffeomorphisms away from $q, q_{j}=0$. This will actually be the case in the first part of our analysis where $\left(u_{1}\right)_{k}^{\sim}$ is assumed to be of compact support. In the notations of next lemma, the lower index of a cone $C$ refers to the paraboloid from which - via a corresponding transform reflected in the upper index - the cone originated. Thus, for $k \neq 0$ and $\Omega_{1}=\Omega_{2}$ the upper index refers to the pertaining transform $(j=1,2$ in (3.3)). For $k=0, j=1$ the meaning is the same (mapping $\Omega_{0}$ via $j=1$ transform) and for the case $k=0, j=2$ the upper index stands for the singular mapping of $\Omega_{0}$ via (3.4).

Lemma 3.3. Coordinate transformations (3.3) map paraboloids $\Omega_{1}=\Omega_{2}$ onto cones

$$
\begin{aligned}
& C_{1}^{1}=C_{2}^{1}=\left\{\left(p, q_{1}\right):|p| \leq \infty, q_{1} \in\left(\sqrt{\left(a_{2}-a_{1}\right) / a_{1}}|p|, \infty\right)\right\}, \\
& C_{1}^{2}=C_{2}^{2}=\left\{\left(p, q_{2}\right):|p| \leq \infty, q_{2} \in(0, \infty)\right\},
\end{aligned}
$$

while transformations (3.3) for $j=1$ and (3.4) respectively map $\Omega_{0}$ onto cones

$$
\begin{aligned}
& C_{0}^{1}=\left\{\left(p, q_{0}^{1}\right):|p| \leq \infty, q_{0}^{1} \in\left(0, \sqrt{\left(a_{2}-a_{1}\right) / a_{1}}|p|\right)\right\}, \\
& C_{0}^{2}=\left\{(p, q):|p| \leq \infty, q \in\left(-\sqrt{\left(a_{2}-a_{1}\right) / a_{2}}|p|, 0\right)\right\} .
\end{aligned}
$$

This transformation is a local diffeomorphism on every compact subset of $\Omega_{k}, k=1,2$.

Proof. We give a proof for the singular case $k=0$ as the rest proceeds in analogy. For $j=1$ we have $\mu=a_{1}\left(|p|^{2}+q_{1}^{2}\right)$. First inequality in the definition of $\Omega_{0}$ implies $a_{1}|p|^{2}<a_{1}\left(|p|^{2}+q_{1}^{2}\right)$ which holds for all $q_{1} \neq 0$. The second inequality implies $a_{1}\left(|p|^{2}+q_{1}^{2}\right)<a_{2}|p|^{2}$. The choice of the positive square root from $q_{1}^{2}$ yields the assertion.

For $(k, j)=(0,2)$ we have $\mu=a_{2}\left(|p|^{2}-q^{2}\right)$ so the first inequality in the definition of $\Omega_{0}$ implies $a_{2} q^{2}<\left(a_{2}-a_{1}\right)|p|^{2}$. Now the negative choice of the square root provides the assertion as the second inequality obviously holds for any $q \neq 0$. 
Next lemma readily follows (with $q \rightarrow-q$ for $(k, j)=(0,2)$ ).

LEMma 3.4. Coordinate transformations (3.3)-(3.4) map the integrals of Lemma 3.2 onto

$$
\begin{aligned}
I_{k}^{j}(t, X) & =\int_{C_{k}^{j}} A_{k}^{j}\left(p, q_{j}\right)\left(u_{1}\right)_{k}^{\sim}\left(p, q_{j}\right) \exp \left[i\left( \pm \sqrt{a_{j}\left(|p|^{2}+q_{j}^{2}\right)} t-p x_{2} \mp q_{j} x\right)\right] d p d q_{j} \\
\text { for }(k, j) & \neq(0,2) \text { with } C_{0}^{1} \text { and } C_{k}^{j} \text { from Lemma 3.3 and for }(k, j)=(0,2) \\
I_{0}^{2}(t, X) & =\int_{C_{0}^{2}} A_{0}^{2}(p, q)\left(u_{1}\right)_{0}^{\sim}(p, q) e^{-q x} \exp \left[i\left( \pm \sqrt{a_{2}\left(|p|^{2}-q^{2}\right)} t-p x_{2}\right)\right] d p d q
\end{aligned}
$$

with

$$
A_{k}^{j}=\frac{B_{k}^{j}\left(|p|, q_{j}\right)}{\sqrt{a_{j}\left(|p|^{2}+q_{j}^{2}\right)}} q_{j}(k, j) \neq(0,2) ; A_{0}^{2}=\frac{B_{0}^{2}(|p|, q)}{\sqrt{a_{2}\left(|p|^{2}-q^{2}\right)}} q .
$$

According to Definition 3.1 whole expressions $A_{k}^{j}\left(p, q_{j}\right)\left(u_{1}\right)_{k}^{\sim}\left(p, q_{j}\right)$ are called amplitudes. If it causes no confusion we occasionally say "amplitude" when referring to $A_{k}^{j}$ alone.

But the crux of the matter is in the phases. As last lemma makes clear, the phase for $(k, j) \neq(0,2)$ involves an inner product of $\left(p, q_{j}\right)$ and the space point $X$. For $(k, j)=(0,2)$, however, third components of $q$ and $x$ do not multiply within the phase but in the argument of a decreasing exponential. This makes a decisive difference in time asymptotic behavior.

Definition 3.5. Integrals $I_{k}^{j}(t, X)$ for $(k, j) \neq(0,2)$ will be called non-degenerate parts of the solution $u(t, X)$ and their phases, denoted by $f_{k}^{j}\left(P_{j}, t, X\right)$, will be called non-degenerate phases. In contrast, $I_{0}^{2}(t, X)$ will be called degenerate part of the solution $u(t, X)$ and its phase, denoted by $f(P, t, X)$, will be called degenerate phase.

Stationary phase techniques require that parameters of oscillating integrals - namely $(t, X)$ - remain inside of some compact set. Following [28] we set

$$
\omega:=\sqrt{t^{2}+|X|^{2}}, S:=\left(s_{0}, s_{2}, s\right) \in \mathrm{S}_{4}^{1}, s_{2}=\left(s_{2}^{1}, s_{2}^{2}\right)
$$

where

$$
\mathrm{S}_{4}^{1}=\left\{S \in \mathbb{R}^{4}: s_{0}^{2}+\left|s_{2}\right|^{2}+s^{2}=1, s_{0} \geq 0,\left|s_{2}\right|^{2} \geq 0, s \geq 0\right\}
$$

so every time-space point is represented as (for unique $S \in \mathrm{S}_{4}^{1}$ )

$$
(t, X)=\left(t, x_{2}, x\right)=\omega\left(s_{0}, s_{2}, s\right)=\omega S=:(\omega, S) .
$$

As we are only interested in large times, we assume $t \geq 1 \Longrightarrow \omega \geq 1$ by (3.8). Thus, the role of parameters is played by $\mathrm{S}$ and $\omega$. Nonetheless, we interchangeably write $I_{k}^{j}(t, X)$ to keep track of natural time-space. Next theorem summarizes considerations of this section. 
Theorem 3.6. Substitutions in (3.8) transform the integrals of Lemma 3.4 into, for $(k, j) \neq(0,2)$

$$
\begin{aligned}
I_{k}^{j}(\omega, S)= & \int_{C_{k}^{j}} A_{k}^{j}\left(p, q_{j}\right)\left(u_{1}\right)_{k}\left(p, q_{j}\right) \\
& \exp \left\{i \omega\left[\left( \pm \sqrt{a_{j}\left(|p|^{2}+q_{j}^{2}\right)} s_{0}-p s_{2} \mp q_{j} s\right)\right]\right\} d p d q_{j}
\end{aligned}
$$

and for $(k, j)=(0,2)$

$$
\begin{aligned}
I_{0}^{2}(\omega, S)= & \int_{C} A_{0}^{2}(p, q)\left(u_{1}\right)_{0}^{\sim}(p, q) e^{-\omega q s} \\
& \exp \left\{i \omega\left[\left( \pm \sqrt{a_{2}\left(|p|^{2}-q^{2}\right)} s_{0}-p s_{2}\right)\right]\right\} d p d q .
\end{aligned}
$$

\section{Degenerate Part on $\tilde{\mathcal{H}}$}

We first estimate $I_{0}^{2}(\omega, S)$ from Theorem 3.6 for large $\omega$; recall also the norm (2.1) from section 2

$$
\|f\|_{L^{1, m}\left(\mathbb{R}_{+}^{3} ; X\right)}=\sum_{|\alpha| \leq m_{\mathbb{R}_{+}^{3}}} \int\left|X^{\alpha} f(X)\right| d X
$$

and set $C=C_{0}^{2}$. We apply a theorem stating that oscillating integrals with no stationary points are rapidly decreasing functions of the large parameter (cf. [13, Theorem 7.7.1]).

TheOREM 4.1. Let $\mathcal{K} \subset \mathbb{R}^{n}$ be a compact set, $\mathcal{X}$ an open neighborhood of $\mathcal{K}$, and $m$ a non-negative integer. If $u \in C_{0}^{m}(\mathcal{X})$ and $\phi$ is a real-valued function over $\mathcal{X}$, then it holds (for all $\lambda>0$ )

$$
\lambda^{m}\left|\int u(\theta) \exp (i \lambda \phi(\theta)) d \theta\right| \leq \text { Const. } \sum_{|\alpha| \leq m} \sup \left|D^{\alpha} u\right|\left|\phi^{\prime}\right|^{|\alpha|-2 m} .
$$

Const. is bounded when $\phi$ stays in a bounded set in $C^{m+1}(\mathcal{X})$. It is a rational function of $\phi^{\prime}$.

The final statement is contained in the proof of Theorem 7.7.1 of [13]. Note that the following subspace of $\mathcal{H}$ introduced via

$$
\tilde{\mathcal{H}}=\left\{f \in \mathcal{H}:(f)_{k} \in C_{0}^{\infty}\left(C_{k}^{j}\right), k=0,1,2, j=1,2\right\}
$$

is dense in $\mathcal{H}$ as the mapping

$$
f \longrightarrow\left((f)_{k}^{\sim}\right)_{k=0,1,2}
$$


is an isometric isomorphism (cf. [6, Lemma 3.9]) of the spaces $\mathcal{H}$ and

$$
\left(\prod_{k=0}^{2} L_{2}\left(\Omega_{k}\right),\left(\sum_{k=0}^{2}\left\|()_{k}^{\sim}\right\|_{L_{2}\left(\Omega_{k}\right)}^{2}\right)^{1 / 2}\right)
$$

which maps $\tilde{\mathcal{H}}$ to $\prod_{k=0}^{2} \mathcal{C}_{0}^{\infty}\left(\Omega_{k}\right)$, the latter being a dense subset in $\prod_{k=0}^{2} L_{2}\left(\Omega_{k}\right)$ and as such consisting of transforms of $\mathcal{H}$ - functions. We first consider initial data $u_{1} \in \tilde{\mathcal{H}}$, then extend results to $u_{1} \in C_{0}^{\infty}$. Under above assumptions it is clear that the amplitudes $A_{0}^{2}(p, q)\left(u_{1}\right)_{0}^{\sim}(p, q)$ of $I_{0}^{2}(\omega, S)$ belong to $\mathcal{C}_{0}^{\infty}(C)$ as the only possible singularity of $A_{0}^{2}(p, \mu)$ is the point $(p, q)=(0,0)$ which is the edge of $C$. Indeed, the same holds for any $(k, j) \in\{0,1,2\} \times\{1,2\}$.

Lemma 4.2. For $\left(u_{1}\right)_{0}^{\sim} \in \mathcal{C}_{0}^{\infty}(C)$ the phase of $I_{0}^{2}(\omega, S)$ given by

$$
f(p, q ; S)= \pm \sqrt{a_{j}\left(|p|^{2}-q^{2}\right)} s_{0}-p s_{2}
$$

is never stationary.

Proof. Denoting the gradient of $f(p, q ; S)$ with respect to the integration variable $P=(p, q)=\left(p_{1}, p_{2}, q\right)$ by $f^{\prime}(P, S)$ we have

$$
f^{\prime}(P ; S)= \pm \sqrt{\frac{a_{2}}{\left(|p|^{2}-q^{2}\right)}} s_{0}\left(p_{1}, p_{2},-q\right)-\left(s_{2}^{1}, s_{2}^{2}, 0\right) .
$$

For this to vanish it must hold (for any choice of $S$-parameters)

$$
\pm \sqrt{\frac{a_{2}}{\left(|p|^{2}-q^{2}\right)}} p=\frac{s_{2}}{s_{0}} \quad \text { and } \quad q=0 .
$$

But the latter cannot hold on $\operatorname{supp}\left(\left(u_{1}\right)_{0}^{\sim}\right) \subset C$, as we wanted to show.

We can now derive an $L^{1}-L^{\infty}$-like estimate using Theorem 4.1.

TheOREM 4.3. For $\left(u_{1}\right)_{0}^{\sim} \in \mathcal{C}_{0}^{\infty}(C)$ the oscillating integral $I_{0}^{2}(t, X)$ is a rapidly decreasing function of the time variable $t$, i.e. $(\forall t>0),\left(\forall X \in K_{2}\right)$, $(\forall m \in N)\left(\exists C_{0}^{2}(m)\right)$ such that

$$
\left|I_{0}^{2}(t, X)\right| \leq C_{0}^{2}(m) t^{-m}\left\|u_{1}\right\|_{L^{1, m}\left(\mathbb{R}_{+}^{3} ; X\right)} .
$$

The constants $C_{0}^{2}(m)$ are given by

$$
\begin{aligned}
C_{0}^{2}(m)= & \text { const. } \max \left\{1, \sum_{l \leq m} \sup \left(\frac{l}{e q}\right)^{l}\right\}\left(\sum_{|\alpha| \leq m} \sup \left|f^{\prime}(P, S)\right|^{|\alpha|-2 m}\right) \\
& \times\left(\sum_{|\alpha| \leq m} \sup \left|D_{P}^{\alpha} A_{0}^{2}(P)\right|\right)\left(\sum_{|\alpha| \leq m} \sup \left|D_{P}^{\alpha} B_{0}^{2}(P)\right|\right),
\end{aligned}
$$

where const. only depends on the coefficients $a_{1}, a_{2}, d_{1}, d_{2}$ and the suprema are taken over supp $\left(\left(u_{1}\right)_{0}^{\sim}\right) \times S_{4}^{1}$. 
Proof. We have shown that Theorem 4.1 can be applied as we can find (due to compactness of supp $\left.\left(u_{1}\right)_{k}^{\sim}\right)$ an open neighborhood $U$ of $\operatorname{supp}\left(u_{1}\right)_{k}$ where the phase is $\mathcal{C}^{\infty}$. By Theorem 3.6 it suffices to show that the estimate holds for $\left|I_{0}^{2}(\omega, S)\right|$. Now, Theorem 4.1 implies that for an arbitrary $m \in N$ there is a $c_{0}^{2}(m)$ such that

$$
\begin{aligned}
& \left|I_{0}^{2}(\omega, S)\right| \\
& \leq c_{0}^{2}(m) \omega^{-m} \sum_{|\alpha| \leq m} \sup \left(\left|f^{\prime}(P, S)\right|^{|\alpha|-2 m}\left|D_{P}^{\alpha}\left[e^{-\omega q s}\left(A_{0}^{2}\left(u_{1}\right)_{0}^{\sim}\right)(P)\right]\right|\right) \\
& \leq c_{0}^{2}(m) \omega^{-m} \sum_{|\alpha| \leq m} \sup \left(\left|f^{\prime}(P, S)\right|^{|\alpha|-2 m}\right. \\
& \left.\quad \sum_{|\alpha| \leq m}\left|D_{P}^{\alpha}\left[e^{-\omega q s}\left(A_{0}^{2}\left(u_{1}\right)_{0}^{\sim}\right)(P)\right]\right|\right)
\end{aligned}
$$

uniformly in $S \in \mathrm{S}_{4}^{1}$. To see uniformity, note that the only function depending on $S$ is the phase function $f$. Noting that $S$-parameters stay in a compact set $\mathrm{S}_{4}^{1}$ and setting $f^{S}(P):=f(P, S)$ we observe that $\left\{f^{S}(\cdot)\right\}_{S \in \mathrm{S}_{4}^{1}}$ is a bounded subset of $\mathrm{C}_{0}^{\infty}(U)$ due to continuity of $f(P, S)$ over $U \times \mathrm{S}_{4}^{1}$. Therefore, $c_{0}^{2}(m)$ does not depend on the $S$ - parameter by Theorem 4.1.

Also, as the derivative of the phase does not vanish, $\left|f^{\prime}(P, S)\right|^{|\alpha|-2 m}$ is continuous as well and uniformity is shown by same arguments. Remaining estimate of the sums involving amplitudes on the right hand side is provided in Proposition 8.3 of the appendix.

\section{Non-Degenerate Parts on $\tilde{\mathcal{H}}$}

Having established that $I_{0}^{2}(t, X)$ is a rapidly decreasing function of time at any space point $X$, we turn to non-degenerate cases $(k, j) \neq(0,2)$ and estimate the integrals $I_{k}^{j}(t, X)$ given by

$$
\int_{C_{k}^{j}} A_{k}^{j}\left(p, q_{j}\right)\left(u_{1}\right)_{k}\left(p, q_{j}\right) \exp \left[i\left( \pm \sqrt{a_{j}\left(|p|^{2}+q_{j}^{2}\right) t}-p x_{2} \mp q_{j} x\right)\right] d p d q_{j} .
$$

Denoting the space part of $S$ by $\tilde{S}$ so that

$$
X=\omega \tilde{S}=\omega\left(s_{2}, s\right)
$$

we first identify two genuinely different classes of space-time points.

THEOREM 5.1. If $(k, j) \neq(0,2)$ the points of stationary phase for the integral $I_{k}^{j}(t, X)$ exist if and only if the relation

$$
|X|=\sqrt{a_{j}} t \Longleftrightarrow|\tilde{S}|=\sqrt{a_{j}} s_{0}
$$

holds for the time-space point $(t, X)$. 
Proof. Phase functions in this case read

$$
f_{j}\left(P_{j}, S\right):=f_{k}^{j}\left(P_{j}, S\right)= \pm \sqrt{a_{j}\left(|p|^{2}+q_{j}^{2}\right)} s_{0}-p s_{2} \mp q_{j} s
$$

with $k$-indices omitted because only $j$-indices make a difference. We calculate the gradient of the phase with respect to $P_{j}$ for both $j=1,2$ to obtain

$$
\begin{aligned}
f_{j}{ }^{\prime}\left(P_{j}, S\right) & = \pm \sqrt{\frac{a_{j}}{|p|^{2}+q_{j}^{2}}} s_{0}\left(p_{1}, p_{2}, q_{j}\right)-\left(s_{2}^{1}, s_{2}^{2}, \pm s\right) \\
& = \pm \sqrt{a_{j}} s_{0} \frac{P_{j}}{\left|P_{j}\right|}-\left(s_{2}, \pm s\right) .
\end{aligned}
$$

With $(5.1),(t, X)=\omega S=\left(\omega s_{0}, \omega \tilde{S}\right)$ and $\tilde{S}_{ \pm}:=\left(s_{2}, \pm s\right)$ we see that the gradient vanishes if and only if

$$
\pm \frac{P_{j}}{\left|P_{j}\right|}=\frac{\tilde{S}_{ \pm}}{\sqrt{a_{j}} s_{0}} \Longleftrightarrow 1=\frac{|X|}{\sqrt{a_{j}} t} .
$$

Therefore, for any time-space point $(t, X)$ with $X \neq \sqrt{a_{j}} t$ there are no stationary points for any of the integrals $I_{k}^{j}(t, X)$. In fact, we have a result analogous to Theorem 4.3.

TheOREM 5.2. For $(k, j) \neq(0,2)$ and $\left(u_{1}\right)_{k} \in \mathcal{C}_{0}^{\infty}\left(C_{k}^{j}\right) \quad \Longleftrightarrow\left(u_{1}\right)_{k}^{\sim} \in$ $\left.\mathcal{C}_{0}^{\infty}\left(\Omega_{k}\right)\right)$, the integral $I_{k}^{j}(t, X)$ is a rapidly decreasing function of the time variable $t$, i.e. $(\forall t>0),\left(\forall X \in K_{j}\right.$ with $\left.|X| \neq \sqrt{a_{j}} t\left(\Longleftrightarrow|\tilde{S}| \neq \sqrt{a_{j}} s_{0}\right)\right),(\forall m \in$ $N)\left(\exists C_{k}^{j}(m)\right)$ such that

$$
\left|I_{k}^{j}(t, X)\right| \leq C_{k}^{j}(m) t^{-m}\left\|u_{1}\right\|_{L^{1, m}\left(\mathbb{R}_{+}^{3} ; X\right)} .
$$

Constants $C_{k}^{j}(m)$ are given by

$$
\begin{aligned}
C_{k}^{j}(m)= & \text { const. }\left(\sum_{|\alpha| \leq m} \max _{j=1,2} \sup \left|\left(f_{j}\right)^{\prime}\left(P_{j}, S\right)\right|^{|\alpha|-2 m}\right) \\
& \left(\sum_{|\alpha| \leq m} \sup \left|D_{P_{j}}^{\alpha} A_{k}^{j}\left(P_{j}\right)\right|\right)\left(\sum_{|\alpha| \leq m} \sup \left|D_{P_{j}}^{\alpha} B_{k}^{j}\left(P_{j}\right)\right|\right) .
\end{aligned}
$$

Suprema are taken over the support of $\left(u_{1}\right)_{k}^{\sim}$. If $\mathcal{S}$ is a compact subset of $S_{4}^{1}$ the estimate is bounded with respect to $S \in \mathcal{S}$ and const only depends on the coefficients $a_{1}, a_{2}, d_{1}, d_{2}$.

Proof. Arguments in line with the proof of Theorem 4.3 show that conditions of Theorem 4.1 are satisfied for amplitudes $\left(A_{k}^{j}\left(u_{1}\right) \tilde{k}\right)\left(p, q_{j}\right), k=0,1,2$ and phases

$$
f_{j}\left(p, q_{j} ; S\right)= \pm \sqrt{a_{j}\left(|p|^{2}+q_{j}^{2}\right)} s_{0}-p s_{2} \mp q_{j}
$$


for any $(k, j) \neq(0,2)$ as we can find (due to compactness of supp $\left(u_{1}\right) \tilde{k}$ ) an open neighborhood $U_{k}^{j}$ of $\operatorname{supp}\left(u_{1}\right)_{k}^{\sim}$ where the phase is $\mathcal{C}^{\infty}$. When the $S$-parameters range over a compact set it follows from Theorem 4.1 that for any $m \in N$ there is a $c_{k}^{j}=c_{k}^{j}(m)$ with

$$
\begin{aligned}
& \left|I_{k}^{j}(t, S)\right| \leq \\
& \quad \leq c_{k}^{j} \omega^{-m} \sum_{|\alpha| \leq m} \sup \left(\left|f_{j}^{\prime}\left(P_{j}\right)\right|^{|\alpha|-2 m}\left|D_{P_{j}}^{\alpha}\left(A_{k}^{j}\left(P_{j}\right)\left(u_{1}\right)_{k}^{\sim}\right)\left(P_{j}\right)\right|\right) \\
& \quad \leq c_{k}^{j} \omega^{-m} \sum_{|\alpha| \leq m} \sup \left|f_{j}^{\prime}\left(P_{j}\right)\right|^{|\alpha|-2 m} \sum_{|\alpha| \leq m}\left|D_{P_{j}}^{\alpha}\left(A_{k}^{j}\left(P_{j}\right)\left(u_{1}\right)_{k}^{\sim}\right)\left(P_{j}\right)\right| .
\end{aligned}
$$

Uniformity with respect to $S$ follows in the same way as in Theorem 4.3. Sums involving amplitudes are estimated in Proposition 8.5 of the appendix.

Obviously, any significant contributions to the solution can only come from the remaining case $(k, j) \neq(0,2)$ and $|X|=\sqrt{a_{j}} t$. Indeed, integrals involved (can) have stationary points. We use [13, Theorem 7.7.1].

TheOREM $5.3([13])$. Let $\mathcal{K} \subset \mathbb{R}^{n}$ be a compact set, $\mathcal{X}$ an open neighborhood of $\mathcal{K}$ and $m$ a positive integer. If $u \in C_{0}^{2 m}(\mathcal{K}), \phi \in C^{3 m+1}(\mathcal{X})$ and $\phi$ is a real valued function over $\mathcal{X}, \phi^{\prime}\left(\theta_{0}\right)=0$, $\operatorname{det} \phi^{\prime \prime}\left(\theta_{0}\right) \neq 0$ and $\phi^{\prime} \neq 0$ in $\mathcal{K} \backslash\left\{\theta_{0}\right\}$, then it holds

$$
\begin{aligned}
& \left|\int u(\theta) \exp (i \lambda \phi(\theta)) d \theta-e^{i \lambda \phi\left(\theta_{0}\right)}\left(\operatorname{det}\left(\lambda \phi^{\prime \prime}\left(\theta_{0}\right) / 2 \pi i\right)\right)^{-1 / 2} \sum_{l<m} \lambda^{-l} L_{l} u\right| \\
& \leq \lambda^{-m} \text { Const. } \sum_{|\alpha| \leq 2 m} \sup \left|D^{\alpha} u\right|, \quad \lambda>0 .
\end{aligned}
$$

Here Const. is bounded when $\phi$ stays in a bounded set in $C^{3 m+1}(\mathcal{X})$. With

$$
g_{\theta_{0}}(\theta):=\phi(\theta)-\phi\left(\theta_{0}\right)-\frac{1}{2}\left(\phi^{\prime \prime}\left(\theta_{0}\right)\left(\theta-\theta_{0}\right), \theta-\theta_{0}\right)
$$

which vanishes of third order at $\theta_{0}$ we have

$$
L_{l} u=\sum_{\nu-\mu=l} \sum_{2 \nu \geq 3 \mu} i^{-l} 2^{-\nu}\left(\phi^{\prime \prime}\left(\theta_{0}\right)^{-1} D, D\right)^{\nu}\left(g_{\theta_{0}}^{\mu} u\right)\left(\theta_{0}\right) / \mu ! \nu ! .
$$

The first term in the expansion on the left hand side of the estimate reads

$$
\lambda^{-\frac{n}{2}} u\left(\theta_{0}\right) e^{i \pi \operatorname{sign}\left(\operatorname{det}\left(\phi^{\prime \prime}\left(\theta_{0}\right)\right)\right) / 4} \frac{2 \pi}{\sqrt{\left|\operatorname{det} \phi^{\prime \prime}\left(\theta_{0}\right)\right|}} \times \exp \left(i \lambda \phi\left(\theta_{0}\right)\right) .
$$

Here $D$ is as in section 2 and the nonnegative integers $\nu, \mu$ denote the numbers of subsequent applications (compositions) of the formal inner products $(D, D)$ and $g$. However, as we are concerned with $L^{1,2}$ - estimates where the second upper index 2 corresponds to taking $m=1$ in (5.2) and with it making 
$|\alpha| \leq 2$ (as will become fully transparent in Theorem 5.7 and its proof) it is only the last formula that matters, as from $m=1$ follows $l=0$ and with this perforce $\mu=0, \nu=0$. Thus, the first term of the expansion on the left hand side is, for our purposes, also the only one; apart from this consideration, we have just taken the complex square root in $\left.\left(\operatorname{det}\left(\lambda \phi^{\prime \prime}\left(\theta_{0}\right) / 2 \pi i\right)\right)^{-1 / 2}\right)$.

The proof of Theorem 5.3 provides another useful point (cf. [13, proof of Theorem 7.7.5 and Lemma 7.7.3]).

Proposition 5.4. The Const. of Theorem 5.3 is of the form

$$
\text { Const. }=C C(u) C(\phi) R\left(\phi^{\prime}\right) \sup \frac{\left|\theta-\theta_{0}\right|^{\kappa}}{\left|\phi^{\prime}(\theta)\right|}
$$

where $\kappa \in \mathbf{N}$, and $C>0$ depends neither on the variable $\theta$ nor on the functions $\phi$, u. Further, $C(h)=\sup \sum_{|\alpha| \leq k}\left|\partial^{\alpha} h(\theta)\right| / \alpha$ ! for $h \in\{\phi, u\}$ and $R\left(\phi^{\prime}\right)$ is a rational function of $\phi^{\prime}$.

We first observe that these results cannot be applied immediately:

TheOREM 5.5. Stationary points of integrals $I_{k}^{j}(\omega, S),(k, j) \neq(0,2)$ lie on the rays $P_{j}(\tilde{S})=\gamma \tilde{S} \Leftrightarrow P_{j}(X)=\gamma X, \gamma>0$. All these points are degenerate.

Proof. With notation of Theorem 5.1, the gradient of the phase with respect to $P_{j}$ reads $f_{j}{ }^{\prime}\left(P_{j}, S\right)= \pm \sqrt{a_{j}} s_{0} \frac{P_{j}}{\left|P_{j}\right|}-\tilde{S}_{ \pm}$. As $\left|\tilde{S}_{ \pm}\right|=\sqrt{a_{j}} s_{0}$ must hold by Theorem 5.1, first assertion follows by direct substitution. The quick observation

$$
\operatorname{det}\left(f_{j}^{\prime \prime}\left(p, q_{j} ; s_{2}\right)\right)=2 p_{1}^{2} p_{2}^{2} q_{j}^{2}-2 p_{1}^{2} p_{2}^{2} q_{j}^{2}=0
$$

completes the proof.

So, as Theorem 5.3 is not directly applicable in three dimensions, we temporarily fix the third integration variable $q_{j}$ and turn to the inner two dimensional integral with respect to $p=\left(p_{1}, p_{2}\right)$. Writing

$$
\begin{aligned}
I_{k}^{j} & (t, X)= \\
= & \int_{C_{k}^{j}}\left(A_{k}^{j}\left(u_{1}\right)_{k}^{\sim}\right)\left(p, q_{j}\right) \exp \left[i \omega\left( \pm \sqrt{a_{j}\left(|p|^{2}+q_{j}^{2}\right)} s_{0}-p s_{2} \mp q_{j} s\right)\right] d p d q_{j} \\
= & \int\left\{\iint\left(A_{k}^{j}\left(u_{1}\right)_{k}^{\sim}\right)\left(p, q_{j}\right) \exp \left[i \omega\left( \pm \sqrt{a_{j}\left(|p|^{2}+q_{j}^{2}\right)} s_{0}-p s_{2}\right] d p\right\}\right. \\
& \times \exp \left[i \omega\left(\mp q_{j} s\right)\right] d q_{j}
\end{aligned}
$$

and letting $I_{k}^{j}\left(\omega, q_{j} ; s_{2}\right)$ denote the inner integral in curly brackets, we apply Theorem 5.3 to $I_{k}^{j}\left(\omega, q_{j} ; s_{2}\right)$. 
Theorem 5.6. For any $q_{j}>0, s_{0}>0$ and fixed $s_{2}$ with $\sqrt{a_{j}} s_{0}>\left|s_{2}\right|$ the integral $I_{k}^{j}\left(\omega, q_{j} ; s_{2}\right)$ has a unique non-degenerate stationary point.

Proof. We observe the integral $I_{k}^{j}\left(\omega, q_{j} ; S\right)$ in the $q_{j}$-cut of $C_{k}^{j}$ for fixed $q_{j}>0$. The gradient of its phase reads

$$
f_{j}{ }^{\prime}\left(p_{1}, p_{2}, q_{j} ; S\right)=\left(\frac{\sqrt{a_{j}} s_{0} p_{1}}{\sqrt{|p|^{2}+q_{j}^{2}}}-s_{2}^{1}, \frac{\sqrt{a_{j}} s_{0} p_{2}}{\sqrt{|p|^{2}+q_{j}^{2}}}-s_{2}^{2}\right)
$$

implying that $\frac{p_{1}^{0}}{p_{2}^{0}}=\frac{s_{2}^{1}}{s_{2}^{2}}$ must hold for a stationary point $p^{0}=\left(p_{1}^{0}, p_{2}^{0}\right)$. Therefore, stationary points are given by

$$
p^{0}=\left(p_{1}^{0}, p_{2}^{0}\right)=\left(\frac{s_{2}^{1} q_{j}}{\sqrt{a_{j} s_{0}^{2}-\left|s_{2}\right|^{2}}}, \frac{s_{2}^{2} q_{j}}{\sqrt{a_{j} s_{0}^{2}-\left|s_{2}\right|^{2}}}\right)=\frac{q_{j}}{\sqrt{a_{j} s_{0}^{2}-\left|s_{2}\right|^{2}}} s_{2} .
$$

It remains to show that each $p^{0}$ is non-degenerate. It is readily verified that

$$
\operatorname{det}\left(f_{j}^{\prime \prime}\left(p, q_{j} ; s_{2}\right)\right)=\frac{a_{j} s_{0}^{2} q_{j}^{2}}{\left(|p|^{2}+q_{j}^{2}\right)^{2}}
$$

at $p^{0}$ and, with $\left|p^{0}\right|^{2}=\left[q_{j}^{2} /\left(a_{j} s_{0}^{2}-\left|s_{2}\right|^{2}\right)\right]\left|s_{2}\right|^{2}$,

$$
\operatorname{det}\left(f_{j}^{\prime \prime}\left(p^{0}, q_{j} ; S\right)\right)=a_{j}^{-1} s_{0}^{-2} q_{j}^{-2}\left(a_{j} s_{0}^{2}-\left|s_{2}\right|^{2}\right)^{2} .
$$

As this cannot vanish under the assumptions, the proof is complete.

Thus Theorem 5.3 is applicable to the inner double integral $I_{k}^{j}\left(\omega, q_{j} ; s_{2}\right)$ with respect to $p$ for any fixed $q_{j}$. Moreover, we obtain the following estimate for the whole three dimensional integral $I_{k}^{j}(t, X)=I_{k}^{j}(\omega, S)$.

TheOrem 5.7. For $(k, j) \neq(0,2)$ and $\left(u_{1}\right)_{k} \in C_{0}^{\infty}\left(C_{k}^{j}\right)$ it holds

$$
\left|I_{k}^{j}(t, X)\right| \leq \text { Const.t }{ }^{-1}\left\|u_{1}\right\|_{L^{1,2}\left(\mathbb{R}_{+}^{3} ; X\right)} .
$$

Here, Const is given by $c c_{k}^{j}$ where $c$ is bounded with respect to the $S$ parameters while $c_{k}^{j}$ is as in Proposition 8.5. The above estimate is optimal in the sense that it cannot hold for any power of $t$ smaller than -1 .

Proof. As $\left(u_{1}\right)_{k} \in C_{0}^{\infty}\left(C_{k}^{j}\right)$ one can choose an $R>0$ with

$$
\operatorname{supp}\left(\left(u_{1}\right)_{k}\right) \subset K(0, R)=\left\{P_{j} \in \mathbb{R}_{P_{j}}:\left|P_{j} \leq R\right|\right\} .
$$

For each $q_{j}$ with $\left(p, q_{j}\right) \in \operatorname{supp}\left(\left(u_{1}\right)_{k}\right)$ denote the $q_{j}$-cut of the ball $K(0, R)$ by $K\left(0, r\left(q_{j}\right)\right)$ (thus $r\left(q_{j}\right)$ denotes the radius of the circle around $\left(0,0, q_{j}\right)$ in the plane $\left\{P_{j}^{\prime}=\left(p^{\prime}, q_{j}^{\prime}\right): q_{j}^{\prime} \equiv q_{j}\right\}$ determined by the $q_{j}$-cut of the ball $\left.K(0, R)\right)$. 
Now recall that, by the last theorem, the stationary points of the integral $I_{k}^{j}\left(\omega, q_{j} ; s_{2}\right)$ are given by

$$
p^{0}=\left(\frac{s_{2}^{1} q_{j}}{\sqrt{a_{j} s_{0}^{2}-\left|s_{2}\right|^{2}}}, \frac{s_{2}^{2} q_{j}}{\sqrt{a_{j} s_{0}^{2}-\left|s_{2}\right|^{2}}}\right)=\frac{q_{j}}{\sqrt{a_{j} s_{0}^{2}-\left|s_{2}\right|^{2}}} s_{2} .
$$

Note that for an arbitrary $s_{0}>0$ and $|\tilde{S}|^{2}=\left|s_{2}\right|^{2}+s^{2}=a_{j} s_{0}^{2}$ it holds

$$
\left|p^{0}\right|=\frac{q_{j}}{\sqrt{a_{j} s_{0}^{2}-\left|s_{2}\right|^{2}}}\left|s_{2}\right| \geq R \Longleftrightarrow\left|s_{2}\right|^{2}=s_{1}^{2}+s_{2}^{2} \geq \frac{R^{2}}{q_{j}^{2}} s^{2} .
$$

On the other hand, if the whole three dimensional integral $I_{k}^{j}(\omega ; S)$ is to have stationary points, the relation

$$
|\tilde{S}|^{2}=\left|s_{2}\right|^{2}+s^{2}=a_{j} s_{0}^{2}
$$

must hold for the $S$-parameter by Theorem 5.1. This, together with

$$
|S|^{2}=\left|s_{2}\right|^{2}+s^{2}+s_{0}^{2}=1,
$$

immediately implies

$$
s_{0}=\text { const. }=\frac{1}{\sqrt{a_{j}+1}}=: s_{0}^{Z} \text { and }|\tilde{S}|^{2}=\left|s_{2}\right|^{2}+s^{2}=\text { const. }=\frac{a_{j}}{a_{j}+1} .
$$

Setting

$$
q_{j}^{m}:=\min \left\{q_{j}:\left(p, q_{j}\right) \in \operatorname{supp}\left(\left(u_{1}\right)_{k}\right)\right\}
$$

and

$$
\left.\begin{array}{c}
\left|s_{2}\right|^{2} \leq \frac{R^{2}}{\left(q_{j}^{m}\right)^{2}} s^{2} \\
\left|s_{2}\right|^{2}+s^{2}=a_{j} s_{0}^{2}, a_{j} s_{0}^{2}-\left|s_{2}\right|^{2}=: s_{R, s_{0}}^{2}>0
\end{array}\right\}
$$

we can define the set

$$
\mathcal{S}_{R, s_{0}}=\left\{\left(s_{0}, s_{2}, s\right) \in S^{4}: s_{2}, s \text { satisfy (5.4) for some } s_{R, s_{0}}>0\right\} .
$$

Making the special choice $s_{0}=s_{0}^{Z}$ we find $\delta_{0}>0, s_{R}>0$ with

$$
a_{j}\left(s_{0}^{Z} \pm \delta\right)^{2}-\left|s_{2}\right|^{2}=s^{2} \geq s_{R}^{2}, \forall \delta \in\left[0, \delta_{0}\right]
$$

and considering the compact segment of the ball $\mathrm{S}_{4}^{1}$ defined by

$$
\mathcal{S}_{R, \delta_{0}}:=\cup_{s_{0} \in\left[s_{0}^{Z}-\delta_{0}, s_{0}^{Z}+\delta_{0}\right]} \mathcal{S}_{R, s_{0}}
$$

as well as the closure of its complement (with respect to $\mathrm{S}_{4}^{1}$ )

$$
\mathcal{S}_{R, \delta_{0}}^{(m)}:=\mathrm{Cl}\left(\mathrm{S}_{4}^{1} \backslash \mathcal{S}_{R, \delta_{0}}\right)
$$

we are left with two cases: 
1) The CASE $S \in \mathcal{S}_{R, \delta_{0}}:$ By construction of $S \in \mathcal{S}_{R, \delta_{0}}$ and Theorem 5.6, the integral $I_{k}^{j}\left(\omega, q_{j} ; s_{2}\right)$ has a unique non-degenerate stationary point for any fixed $q_{j}$ given by

$$
p^{0}=\frac{q_{j}}{\sqrt{a_{j} s_{0}^{2}-\left|s_{2}\right|^{2}}} s_{2} .
$$

For an arbitrary choice of $S \in \mathcal{S}_{R, \delta_{0}}$ Theorem 5.3 gives the estimate (conditions are checked in Theorem 5.6)

$$
\begin{aligned}
& \mid I_{k}^{j}\left(\omega, q_{j} ; s_{2}\right)-\omega^{-1}\left(A_{k}^{j}\left(u_{1}\right)_{k}^{\sim}\right)\left(p^{0}, q_{j}\right) \mathrm{e}^{i \pi \operatorname{sign}\left(\operatorname{det}\left(f_{j}^{\prime \prime}\left(p^{0}, q_{j}\right)\right)\right) / 4} \\
& \quad \frac{2 \pi}{\sqrt{\left|\operatorname{det} f_{j}^{\prime \prime}\left(p^{0}, q_{j}\right)\right|}} \exp \left[i \omega\left(\sqrt{a_{j}\left(\left(p^{0}\right)^{2}+q_{j}^{2}\right)} \mp p^{0} s_{2}\right)\right] \mid \\
& \quad \leq c \omega^{-1} \sum_{|\alpha| \leq 2} \sup \left|D_{P_{j}}^{\alpha}\left(A_{k}^{j}\left(u_{1}\right)_{k}\right)\left(p, q_{j}\right)\right| \leq C t^{-1}\left\|u_{1}\right\|_{L^{1,2}\left(\mathbb{R}_{+}^{3} ; X\right)} .
\end{aligned}
$$

Last inequality follows from Proposition 8.5 of the appendix. The suprema are taken over the support of $\left(u_{1}\right)_{k}$. Setting

$$
F\left(p^{0}, q_{j}\right)=F\left(p^{0}\left(q_{j} ; S\right), q_{j}\right)=\mathrm{e}^{i \pi \operatorname{sign}\left(\operatorname{det}\left(f_{j}^{\prime \prime}\left(p^{0}, q_{j}\right)\right)\right) / 4} \frac{2 \pi}{\sqrt{\left|\operatorname{det} f_{j}^{\prime \prime}\left(p^{0}, q_{j}\right)\right|}}
$$

it is clear that $F\left(p^{0}, q_{j}\right)$ is a continuous (and thus bounded) function on the compact set $\left[q_{j}^{m}, q_{j}^{M}\right] \times \mathcal{S}_{R, \delta_{0}}$ (cf. Theorem 5.6 or the very end of its proof).

We want to show that the estimate is uniform on the parameter set $\mathcal{S}_{R, \delta_{0}}$. This also follows from Theorem 5.3. Indeed, by the latter theorem the constant $c$ in the first inequality of the above estimate remains bounded if the phase function stays in some bounded subset of $C\left(K\left(0, r\left(q_{j}\right)\right)\right)$. But the latter is true in our case since the phase $f_{j}\left(p, q_{j} ; s_{0}, s_{2}\right)$ is a continuous function on the compact set $K\left(0, r\left(q_{j}\right)\right) \times \mathcal{S}_{R, \delta_{0}}$; therefore the set

$$
\left\{f_{j}^{(S)}\left(p, q_{j}\right):=f_{j}\left(p, q_{j} ; s_{0}, s_{2}\right)\right\}_{S \in \mathcal{S}_{R, \delta_{0}}}
$$

is bounded in $C\left(K\left(0, r\left(q_{j}\right)\right)\right)$.

Uniformity of last estimate on $S \in \mathcal{S}_{R, \delta_{0}}$ allows us to occasionally omit the $S$-parameter dependence of the integrals as it does not affect the estimates in terms of modulus. Recalling that

$$
I_{k}^{j}(\omega)=\int_{q_{j}^{m}}^{q_{j}^{M}} I_{k}^{j}\left(\omega, q_{j}\right) \exp \left(\mp i \omega q_{j} s\right) d q_{j}
$$


and writing

$$
\begin{aligned}
I_{k}^{j}\left(\omega, q_{j}\right)= & \omega^{-1} A_{k}^{j}\left(p^{0}, q_{j}\right)\left(u_{1}\right) \underset{k}{\sim}\left(p^{0}, q_{j}\right) F\left(p^{0}, q_{j}\right) \\
& \times \exp \left[i \omega\left(\sqrt{a_{j}\left(\left(p^{0}\right)^{2}+q_{j}^{2}\right)} \mp p^{0} s_{2}\right)\right] \mathrm{e}^{\mp i \omega q_{j} s} \\
& +I_{k}^{j}\left(\omega, q_{j}\right)-\omega^{-1} A_{k}^{j}\left(p^{0}, q_{j}\right)\left(u_{1}\right) \tilde{k}_{k}^{\sim}\left(p^{0}, q_{j}\right) F\left(p^{0}, q_{j}\right) \\
& \times \exp \left[i \omega\left(\sqrt{a_{j}\left(\left(p^{0}\right)^{2}+q_{j}^{2}\right)} \mp p^{0} s_{2}\right)\right] \mathrm{e}^{\mp i \omega q_{j} s}
\end{aligned}
$$

where $F\left(p^{0}, q_{j}\right)$ is defined as in $(5.7)$ we obtain

$$
\begin{aligned}
I_{k}^{j}(\omega)= & \int_{q_{j}^{m}}^{q_{j}^{M}} I_{k}^{j}\left(\omega, q_{j}\right) \exp \left(\mp i \omega q_{j} s\right) d q_{j} \\
= & \omega^{-1} \int_{q_{j}^{m}}^{q_{j}^{M}} A_{k}^{j}\left(p^{0}, q_{j}\right)\left(u_{1}\right)_{k}^{\sim}\left(p^{0}, q_{j}\right) F\left(p^{0}, q_{j}\right) \\
& \times \exp \left[i \omega\left(\sqrt{a_{j}\left(\left(p^{0}\right)^{2}+q_{j}^{2}\right)} \mp p^{0} s_{2}\right)\right] \exp \left(\mp i \omega q_{j} s\right) d q_{j} \\
& +\int_{q_{j}^{m}}^{q_{j}^{M}}\left\{I_{k}^{j}\left(\omega, q_{j}\right)-\omega^{-1} A_{k}^{j}\left(p^{0}, q_{j}\right)\left(u_{1}\right)_{k}^{\sim}\left(p^{0}, q_{j}\right) F\left(p^{0}, q_{j}\right)\right. \\
& \left.\times \exp \left[i \omega\left(\sqrt{a_{j}\left(\left(p^{0}\right)^{2}+q_{j}^{2}\right)} \mp p^{0} s_{2}\right)\right]\right\} \exp \left(\mp i \omega q_{j} s\right) d q_{j} .
\end{aligned}
$$

The modulus of the second integrand has just been estimated in (5.6) so that the modulus of the second integral is dominated by

$$
C t^{-1}\left(q_{j}^{M}-q_{j}^{m}\right)\left\|u_{1}\right\|_{L^{1,2}\left(\mathbb{R}_{+}^{3} ; X\right)} .
$$

The modulus of the first integral is immediately seen to be dominated by

$$
\tilde{C}\left(q_{j}^{M}-q_{j}^{m}\right)\left\|u_{1}\right\|_{L^{1,2}\left(\mathbb{R}_{+}^{3} ; X\right)}
$$

with

$$
\tilde{C}=\sup \left|A\left(p, q_{j}\right) F\left(p, q_{j}\right)\right|
$$

where the supremum is taken over $\operatorname{supp}\left(u_{1}\right)_{k}$. This proves the first part of the theorem in this case.

Optimality: The estimate of $I_{k}^{j}(\omega)$ is sharp due to Theorem 5.3. So it suffices to show that the first term in the last formula cannot be estimated better than by $t^{-1}$. The phase of the latter integral is given by

$$
f_{j}\left(p^{0}\left(q_{j}\right), q_{j} ; s_{0}, s_{2}\right)=\sqrt{a_{j}\left(\left(p^{0}\left(q_{j}\right)\right)^{2}+q_{j}^{2}\right)} \mp p^{0}\left(q_{j}\right) s_{2} \mp q_{j} s
$$


which, calculated at the stationary point $p^{0}\left(q_{j} ; s_{0}, s_{2}\right)$ from (5.5) yields

$$
\begin{aligned}
f_{j}\left(p^{0}\left(q_{j}\right), q_{j} ; s_{0}, s_{2}\right) & =\sqrt{a_{j} \frac{a_{j} s_{0}^{2} q_{j}^{2}}{a_{j} s_{0}^{2}-\left|s_{2}\right|^{2}}} s_{0}-\frac{q_{j}}{\sqrt{a_{j} s_{0}^{2}-\left|s_{2}\right|^{2}}}\left|s_{2}\right|^{2} \mp q_{j} s \\
& =\frac{q_{j}}{\sqrt{a_{j} s_{0}^{2}-\left|s_{2}\right|^{2}}}\left\{a_{j} s_{0}^{2}-\left|s_{2}\right|^{2} \mp s \sqrt{a_{j} s_{0}^{2}-\left|s_{2}\right|^{2}}\right\} .
\end{aligned}
$$

Now remember that by Theorem 5.1 the stationary points of the three dimensional integral $I_{k}^{j}(\omega, S)$ exist if and only if $|X|=\sqrt{a_{j}} t \Longleftrightarrow\left|s_{2}\right|^{2}+s^{2}=a_{j} s_{0}^{2}$ i.e. $s=\sqrt{a_{j} s_{0}^{2}-\left|s_{2}\right|^{2}}$ must hold. For this particular choice of parameters (contained in $\mathcal{S}_{R, \delta}^{r}$ by construction) we have

$$
f_{j}\left(p^{0}\left(q_{j}\right), q_{j} ; s_{0}, s_{2}\right)=a_{j} s_{0}^{2}-\left|s_{2}\right|^{2} \mp a_{j} s_{0}^{2} \pm\left|s_{2}\right|^{2} .
$$

Hence, for an appropriate choice of signs, the phase of the first integral in the above estimate vanishes. Note that this happens exactly at the points of stationary phase of the integral $I_{k}^{j}(\omega, S)$ as it should be - otherwise the integral would be a rapidly decreasing function of $\omega$ by Theorem 4.1. But at the points of vanishing phase the considered integral is not a function of $\omega$; in particular, it is no oscillating function of $\omega$ and thus the performed $t^{-1}$ estimate is optimal.

2) The CASE $S \in \mathcal{S}_{R, \delta_{0}}^{(m)}$ : Note that in this case, by construction, there are no points of stationary phase for the integral $I_{k}^{j}(\omega, S)$ so that Theorem 5.2 applies. Choosing Const. to be the maximum of the constants appearing in estimates given in steps 1) - 2) we conclude the proof of the theorem.

\section{Wave Equations on $\tilde{\mathcal{H}}$}

Theorems 4.3 and 5.7 handled degenerate and non-degenerate solution integrals. Recalling the dense subspace of $\mathcal{H}$ defined by

$$
\tilde{\mathcal{H}}=\left\{f \in \mathcal{H}:(f)_{k} \in C_{0}^{\infty}\left(C_{k}^{j}\right), k=0,1,2, j=1,2\right\}
$$

we can combine both theorems for an overall estimate.

Theorem 6.1. If $u_{0} \equiv 0, u_{1} \in \tilde{\mathcal{H}}$ then there is a Const. $>0$ such that the estimate

$$
\|u\|_{\infty} \leq \text { Const. }{ }^{-1}\left\|u_{1}\right\|_{L^{1,2}\left(\mathbb{R}_{+}^{3} ; X\right)}
$$

holds for the solution $u(t, X)$ of Theorem 2.6. This estimate is optimal (in the sense of Theorem 5.7) and Const-dependencies are as in Theorems 4.3 and 5.7 .

Procedures from sections 3 through 5 can be applied to wave equation in one space dimension with a constant potential in a straightforward manner. 
THEOREM 6.2. The solution of the initial value problem from Definition 2.4 given in Theorem 2.5 can be written as

$$
u(t, x)=\sum_{(k, j), \pm} \alpha_{k}^{j, \pm} I_{k}^{j, \pm}(t, x),(k, j) \in\{(0,1,2)\} \times\{(1,2)\}
$$

where $\alpha_{k}^{j}$ are some complex constants depending only on $a_{1}, a_{2}, d_{1}, d_{2}$. For $(k, j) \neq(0,2)$ it holds

$$
I_{k}^{j}(t, X)=\int_{C_{k}^{j, 1}} A_{k}^{j}\left(q_{j}\right)\left(u_{1}\right)_{k}^{\sim}\left(q_{j}\right) \exp \left[i\left( \pm \sqrt{\left.c_{j}+a_{j} q_{j}^{2}\right)} t \mp q_{j} x\right)\right] d q_{j}
$$

and for $(k, j)=(0,2)$ we have

$$
\left.I_{0}^{2}(t, x)=\int_{C_{0}^{2,1}} A_{0}^{2}(q)\left(u_{1}\right)_{0}^{\sim}(q) e^{-q x} \exp \left[i\left( \pm \sqrt{c_{2}-a_{2} q^{2}}\right) t\right)\right] d q,
$$

that is, with

$$
(t, x)=: \omega\left(s_{0}, s\right)=\omega S, S \in S_{2}^{1}=\left\{S \in \mathbb{R}^{2}: s_{0}^{2}+s^{2}=1, s_{0} \geq 0, s \geq 0\right\}
$$

as in Theorem 3.6, for $(k, j) \neq(0,2)$

$$
I_{k}^{j}(t, x)=\int_{C_{k}^{j, 1}} A_{k}^{j}\left(q_{j}\right)\left(u_{1}\right)_{k}^{\sim}\left(q_{j}\right) \exp \left[i \omega\left( \pm \sqrt{c_{j}+a_{j} q_{j}^{2}} s_{0} \mp q_{j} s\right)\right] d q_{j}
$$

and for $(k, j)=(0,2)$ we have

$$
I_{0}^{2}(t, x)=\int_{C_{0}^{2,1}} A_{0}^{2}(q)\left(u_{1}\right)_{0}^{\sim}(q) e^{-\omega q s} \exp \left[i \omega\left( \pm \sqrt{\left.c_{2}-a_{2} q^{2}\right)} s_{0}\right)\right] d q
$$

Here, for $k=1,2$

$$
C_{k}^{1,1}=: C_{1}^{1}=\left(\sqrt{\frac{c_{2}-c_{1}}{a_{1}}}, \infty\right), C_{k}^{2,1}=: C_{2}^{1}=(0, \infty)
$$

and for $k=0$

$$
C_{0}^{1,1}=\left(0, \sqrt{\frac{c_{2}-c_{1}}{a_{1}}}\right), C_{0}^{2,1}=\left(0, \sqrt{\frac{c_{2}-c_{1}}{a_{2}}}\right) .
$$

The amplitudes $A_{k}^{j}$ are determined from Theorem 2.5 in full analogy with three dimensional discussion (Lemmas 3.2 - 3.4, Theorem 3.6).

Next step is to reduce observations to the space

$$
\tilde{\mathcal{H}}^{1}=\left\{f \in \mathcal{H}^{1}:(f)_{k}^{\sim} \in \mathcal{C}_{0}^{\infty}\left(C_{k}^{j, 1}\right), j=1,2, k=0,1,2\right\}
$$


and initial conditions

$$
u_{1} \in \tilde{\mathcal{H}}^{1} \cap \prod_{j=1}^{2} L^{1,2}\left(N_{j}, x\right)=\tilde{\mathcal{H}}^{1} \cap L^{1,2}(\mathbb{R} ; x)
$$

with the norm

$$
\left\|u_{1}\right\|_{L^{1, m}(R ; x)}=\sum_{\alpha \leq m} \int_{R}\left|x^{\alpha} u_{1}(x)\right| d x, \alpha, m \in N .
$$

We first handle the case $(k, j) \neq(0,2)$. Let $\left(f^{1}\right)_{k}^{j}$ denote the phase function

$$
\left(f^{1}\right)_{k}^{j}\left(q_{j}, S\right)= \pm \sqrt{c_{j}+a_{j} q_{j}^{2}} s_{0} \mp q_{j} s .
$$

For its derivative with respect to $q_{j}$ we have

which vanishes if and only if

$$
\left[\left(f^{1}\right)_{k}^{j}\right]^{\prime}\left(q_{j}, S\right)=\frac{ \pm a_{j} q_{j} s_{0}}{\sqrt{c_{j}+a_{j} q_{j}^{2}}} \mp s
$$

$$
a_{j} q_{j}^{2}\left(a_{j} s_{0}^{2}-s^{2}\right)=c_{j} s^{2}
$$

meaning that the stationary points are uniquely determined by

$$
q_{j}^{0}=\sqrt{\frac{c_{j}}{a_{j}}} \frac{s}{\sqrt{a_{j} s_{0}^{2}-s^{2}}}
$$

for such parameters $S=\left(s_{0}, s\right)$ for which

$$
a_{j} s_{0}^{2}-s^{2}>0 \Leftrightarrow \sqrt{a_{j}} t>x
$$

holds. Noting that

$$
\left[\left(f^{1}\right)_{k}^{j}\right]^{\prime \prime}\left(q_{j}, S\right)= \pm a_{j} s_{0} \frac{c_{j}}{\left(c_{j}+a_{j} q_{j}^{2}\right)^{3 / 2}}
$$

never vanishes for $s_{0}>0$ we see that these stationary points are nondegenerate. We now take $q_{j}^{m}, q_{j}^{M}$ with

$$
\operatorname{supp}\left(u_{1}\right)_{k}^{\sim} \subset\left[q_{j}^{m}, q_{j}^{M}\right]
$$

and note that there are no stationary points in $\operatorname{supp}\left(u_{1}\right)_{k}$ if

i.e., as is readily seen,

$$
\frac{c_{j}}{a_{j}} \frac{s^{2}}{a_{j} s_{0}^{2}-s^{2}} \geq\left(q_{j}^{M}+1\right)^{2}=: R^{2},
$$

$$
s_{0} \leq \frac{\sqrt{c_{j}+a_{j} R^{2}}}{a_{j} R} s .
$$

This means that in this case Theorem 4.1 applies and the estimate

$$
\left|I_{k}^{j}(t, x)\right| \leq \text { const }_{k}^{j}(m) t^{-m}\left\|u_{1}\right\|_{L^{1, m}(R ; x)}
$$

holds for any $m \in N$ with const $_{k}^{j}(m)$ as in Theorem 5.2. 
On the other hand, Theorem 5.3 applies for $S$-parameters satisfying

$$
\left.\begin{array}{c}
a_{j} s_{0}-s>0, s, s_{0} \geq 0 \\
s_{0}^{2}+s^{2}=1 \\
s_{0} \geq \frac{\sqrt{c_{j}+a_{j} R^{2}}}{a_{j} R} s
\end{array}\right\}=: \mathcal{S}_{R}^{1}
$$

in which case we obtain $(1 \leq t \leq \omega)$

$$
\begin{aligned}
\left|I_{k}^{j}(t, x)\right| \leq & \sqrt{\frac{2 \pi}{\left|\left[\left(f_{k}^{j}\right)^{1}\right]^{\prime \prime}\left(q_{j}^{0}\right)\right|}} \omega^{-\frac{1}{2}}\left|\left(A_{k}^{j}\left(u_{1}\right) \tilde{k}\right)\left(q_{j}^{0}\right)\right| \\
& + \text { Const. } \overbrace{\omega^{-1}}^{\leq \omega^{-\frac{1}{2}}} \sum_{\alpha \leq 2} \sup \left|D^{\alpha}\left(A_{k}^{j}\left(u_{1}\right)_{k}\right)\right|
\end{aligned}
$$

as in Theorems 5.6, 5.7. As usual, suprema are taken over $\operatorname{supp}\left(\left(u_{1}\right)_{k}\right)$.

In the case $(k, j)=(0,2)$ we have

$$
\left(f^{1}\right)_{0}^{2}\left(q_{j}, S\right)= \pm \sqrt{c_{j}-a_{j} q_{j}^{2}} s_{0} \text { and }\left[\left(f^{1}\right)_{0}^{2}\right]^{\prime}\left(q_{j}, S\right)=\frac{\mp a_{j} q_{j} s_{0}}{\sqrt{c_{j}-a_{j} q_{j}^{2}}}=0 \Leftrightarrow q_{j}=0
$$

which does not happen for $\operatorname{supp}\left(u_{1}\right)_{0}^{\sim} \subset\left(0, \sqrt{\frac{c_{2}-c_{1}}{a_{2}}}\right)$. Consequently (cf. Theorem 4.3), it holds

$$
\left|I_{0}^{2}(t, x)\right| \leq \operatorname{const}_{0}^{2}(m) t^{-m}\left\|u_{1}\right\|_{L^{1, m}(R ; x)}
$$

for this part of the solution as well. We have shown:

Theorem 6.3. If $u_{0} \equiv 0, u_{1} \in \tilde{\mathcal{H}}^{1}$ then there is a Const. $>0$ such that the estimate

$$
\|u\|_{\infty} \leq \text { Const. } t^{-\frac{1}{2}}\left\|u_{1}\right\|_{L^{1,2}(R ; x)}
$$

holds for the solution $u(t, x)$ of the initial value problem for the wave equation in one space dimension with a constant potential. This estimate is optimal in the sense of Theorem 5.7 and Const - dependencies are as in 6.1.

In our final section we extend the results obtained so far to the physically interesting function space $\mathcal{C}_{0}^{\infty}$.

\section{Extension to test elements}

We start with the one dimensional case. Let $\mathcal{S}$ denote the Schwartz' space of rapidly decreasing functions (e.g. [25], $\mathbf{Z}_{+}^{n}$ are positive integers)

$$
\mathcal{S}\left(\mathbb{R}^{n}\right)=\left\{f \in \mathcal{C}^{\infty}\left(\mathbb{R}^{n}\right):\|f\|_{\alpha, \beta}<\infty \quad \forall \alpha, \beta \in \mathbf{Z}_{+}^{n}\right\}
$$

where $\alpha, \beta$ are multiindices as in section 2 and

$$
\|f\|_{\alpha, \beta}=\sup _{x \in \mathbb{R}^{n}}\left|x^{\alpha} D^{\beta} f(x)\right| .
$$


For restrictions of $\mathcal{S}$ to domains like integration areas $C_{k}^{j, 1}$ from Theorem 6.2 we utilize the following property of the generalized integral transformations $f_{k}^{\sim}$ introduced in Theorem 2.5 due to [4, Theorem 5.2]:

Theorem 7.1. If $f \in \prod_{j=1}^{2} \mathcal{C}_{0}^{\infty}\left(N_{j}\right)$ then $f_{k}^{\sim} \in \mathcal{S}\left(C_{k}^{j, 1}\right)$.

Let from now on $u_{1} \in \prod_{j=1}^{2} \mathcal{C}_{0}^{\infty}\left(N_{j}\right)$ and consider the case $(k, j) \neq(0,2)$. Take the open cover $\left\{K^{1}(0, N)\right\}_{n \in \mathbf{N}}$ of $\mathbb{R}_{q}((K(0, N)$ is a ball around the origin in $\mathbb{R}^{n}$ of radius $N$, the upper index 1 is for a one dimensional interval in $\mathbb{R})$. For any $M \in \mathbf{N}$ consider the closed interval $K^{1}(0, M)$ and the open interval $K^{1}(0, M+1)$ around it. By [13, chapter 1.4, Theorem 1.4.1] we can find a $\phi_{M} \in \mathcal{C}_{0}^{\infty}(K(0, M+1))$ such that $0 \leq \phi \leq 1$ on $K^{1}(0, M+1)$ and $\phi \equiv 1$ holds on a neighborhood of $K^{1}(0, M)$.

Now it is clear that $u^{M}:=\left(u_{1}\right)_{k} \phi^{M} \longrightarrow\left(u_{1}\right)_{k} \sim$ point-wise and the formula (6.1) applies to $I_{k}^{j, M}(t, x)$ denoting the integral $I_{k}^{j}(t, x)$ with $u^{M}$ instead of $\left(u_{1}\right)_{k}$. From (6.1) it follows

$$
\begin{aligned}
\left|I_{k}^{j, M}(t, x)\right| \leq & \omega^{-\frac{1}{2}} \int \frac{\sqrt{2 \pi}}{\left.\mid\left[\left(f^{1}\right)_{k}^{j}\right)\right]^{\prime \prime}\left(q_{j}\right) \mid}\left|A_{k}^{j}\left(q_{j}\right) u^{M}\left(q_{j}\right)\right| d q_{j} \\
& +\omega^{-\frac{1}{2}} \sum_{\alpha \leq 2} \int C\left(u,\left(f^{1}\right)_{k}^{j}\right)\left|D^{\alpha}\left(A_{k}^{j} u^{M}\right)\left(q_{j}\right)\right| d q_{j}
\end{aligned}
$$

where the integrals are over the whole space (which is the same as to take them over $K^{1}(0, M) \cap C_{k}^{j, 1}$ by construction). We want to let $M \rightarrow \infty$ since in that case by the Levi's monotone convergence theorem it follows

$$
I_{k}^{j, M}(t, x) \longrightarrow I_{k}^{j}(t, x)
$$

a.e. if $\lim _{M \longrightarrow \infty} I_{k}^{j, M}$ exists. Due to Theorem 7.1 we readily see that the latter is true if all the "weights" (functions multiplying $\left(u_{1}\right)_{k}$ in (6.1)) are powers of rational functions of $q_{j}$. This demand is obviously fulfilled for $A_{k}^{j}$ as well as for powers of rational functions of $\left(f^{1}\right)_{k}^{j}$ and its derivatives.

Hence the first integral in (6.1) converges and it remains to discuss the behavior of $C\left(u^{M},\left(f^{1}\right)_{k}^{j}\right)$ appearing in the second integral. But this follows from Theorem 4.1 and Proposition 5.4. Same arguments hold in the cases where no stationary points exist $\left((k, j)=(0,2)\right.$ and $\left.\sqrt{a_{j}} t<x\right)$ and Theorem 4.1 suffices. Thus the following extension of Theorem 6.3 holds.

Theorem 7.2. If $u_{0} \equiv 0, u_{1} \in \prod_{j=1}^{2} \mathcal{C}_{0}^{\infty}\left(N_{j}\right)$ then there is a Const. $>0$ such that the estimate

$$
\|u\|_{\infty} \leq \text { Const.C }\left(u_{1}\right) t^{-\frac{1}{2}}
$$


holds for the solution $u(t, x)$ of the initial value problem for the wave equation in one space dimension with a constant potential from Theorem 2.5. This estimate is optimal in the sense of Theorem 6.1. The number Const. depends only on $a_{1}, a_{2}, d_{1}, d_{2}$ and $C\left(u_{1}\right)$ follows from (7.1) (see also Proposition 5.4).

Noting that the proof of Theorem 5.7 is based on formulas similar to (6.1), (5.8)-(5.9) we see that an analogous procedure as in the one-dimensional case considered above leads to the desired result in the three dimensional case for $(k, j) \neq(0,2)$ as well. It is also clear that the same holds for $(k, j)=(0,2)$ in which case (cf. Theorem 4.3) we only need to consider the constant appearing in Theorem 4.1.

Theorem 7.3. If $u_{0} \equiv 0, u_{1} \in \prod_{j=1}^{2} \mathcal{C}_{0}^{\infty}\left(K_{j}\right)$ then there is a Const. $>0$ such that the estimate

$$
\|u\|_{\infty} \leq \text { Const.C }\left(u_{1}\right) t^{-1}
$$

holds for the solution $u(t, X)$ of wave equation in wedges defined in the statement of Theorem 2.6. This estimate is optimal in the sense of Theorem 6.1. The number Const. depends only on $a_{1}, a_{2}, d_{1}, d_{2}$ and $C\left(u_{1}\right)$ follows from the three dimensional version of (7.1) given by (5.8)-(5.9) (see also Proposition 5.4).

\section{Appendix}

In this appendix we give proofs and calculations of some results used in the preceding sections. Suprema are taken over $\operatorname{supp}\left(\left(u_{1}\right)_{k}\right)$ throughout the section.

Lemma 8.1. For any multi-index $\alpha=\left(\alpha_{1}, \alpha_{2}, \alpha_{3}\right)$ and $m \in \mathbf{N}$ it holds

$$
\sum_{|\alpha| \leq m} \sup \left|D_{P}^{\alpha}\left(u_{1}\right)_{0}^{\tilde{0}}\right| \leq \text { const. } \sum_{|\alpha| \leq m} \sup \left|D_{P}^{\alpha} B_{0}^{2}(P)\right|\left\|u_{1}\right\|_{L^{1, m}\left(\mathbb{R}_{+}^{3} ; X\right)} .
$$

The number const. depends only on $a_{1}, a_{2}, d_{1}, d_{2}$.

Proof. Recalling that $\left(u_{1}\right)_{0}^{\sim}(P)$ is a linear combination (cf. 3 ) of the terms

$$
\int_{K_{1}} \frac{d_{1}}{a_{1}} B_{0}^{1}(P) \exp \left( \pm i q_{1} x\right) \exp \left(-i p_{1} y-i p_{2} z\right) u_{1}(X) d X
$$

and

$$
\left.\int_{K_{2}} \frac{d_{2}}{a_{2}} B_{0}^{2}(P) \exp (-q x) \exp \left(-i p_{1} y-i p_{2} z\right)\right) u_{1}(X) d X
$$

the estimate readily follows after an application of $D_{P}^{\alpha}$ and the definition of the $L^{1, m}-$ norm. 
Lemma 8.2. For any $l \in \mathbf{N}$ and $q>0, x>0$ it holds

$$
x^{l} e^{-q x} \leq\left(\frac{l}{q e}\right)^{l} \text {. }
$$

Proof. We have

$$
\left(x^{l} e^{-q x}\right)^{\prime}=x^{l-1}(l-q x) e^{-q x} .
$$

This expression vanishes if and only if $x=\frac{l}{q}$. Since

$$
\begin{aligned}
& \left(x^{l} e^{-q x}\right)^{\prime}>0, x<\frac{l}{q}, \\
& \left(x^{l} e^{-q x}\right)^{\prime}<0, x>\frac{l}{q}
\end{aligned}
$$

we see that the function $x^{l} e^{-q x}$ has its maximum at the point $x=\frac{l}{q}$. But this maximum is $\left(\frac{l}{q e}\right)^{l}$.

Proposition 8.3 now follows by the same arguments as Lemma 8.1 with an additional application of Lemma 8.2:

Proposition 8.3. For any $m \in \mathbf{N},|\alpha| \leq m, x>0$ it holds

$$
\begin{aligned}
& \sum_{|\alpha| \leq m} \sup \left|D_{P}^{\alpha}\left[e^{-\omega x}\left(A_{0}^{2}(P)\left(u_{1}\right)_{0}^{\sim}\right)(P)\right]\right| \leq c_{0}^{2}\left\|u_{1}\right\|_{L^{1, m}\left(\mathbb{R}_{+}^{3} ; X\right)}, \\
& c_{0}^{2}(m)=\text { const. } \max \left\{1, \sum_{l \leq m} \sup \left(\frac{l}{e q}\right)^{l}\right\} \\
& \quad \times\left(\sum_{|\alpha| \leq m} \sup \left|D_{P}^{\alpha} A_{0}^{2}(P)\right|\right)\left(\sum_{|\alpha| \leq m} \sup \left|D_{P}^{\alpha} B_{0}^{2}(P)\right|\right)
\end{aligned}
$$

and const. is a number depending only on the coefficients $a_{1}, a_{2}, d_{1}, d_{2}$.

These results are needed for the discussions of the degenerate part of the solution. The following results regarding the non-degenerate parts follow in the same way; this time, we only need to recall that $\left(u_{1}\right)_{k}\left(P_{j}\right)$ is a linear combination of the terms

$$
\int_{K_{1}} \frac{d_{1}}{a_{1}} B_{k}^{1}\left(P_{j}\right) \exp \left( \pm i q_{1} x\right) \exp \left(-i p_{1} y-i p_{2} z\right) u_{1}(X) d X
$$

and

$$
\left.\int_{K_{2}} \frac{d_{2}}{a_{2}} B_{0}^{2}\left(P_{j}\right) \exp \left( \pm i q_{2} x\right) \exp \left(-i p_{1} y-i p_{2} z\right)\right) u_{1}(X) d X .
$$

LEMMA 8.4. For any multi-index $\alpha=\left(\alpha_{1}, \alpha_{2}, \alpha_{3}\right)$ and $m \in \mathbf{N},|\alpha| \leq m$ it holds

$$
\sum_{|\alpha| \leq m} \sup \left|D_{P_{j}}^{\alpha}\left(u_{1}\right)_{k}^{\sim}\right| \leq \text { const. } \sum_{(l, j)} \sum_{|\alpha| \leq m} \sup \left|D_{P_{j}}^{\alpha} B_{0}^{2}\left(P_{j}\right)\right|\left\|u_{1}\right\|_{L^{1, m}\left(\mathbb{R}_{+}^{3} ; X\right)}
$$


where the first sum is over $(l, j) \in\{(0,1,2) \times(1,2)\}$ and const. is a number depending only on the coefficients $a_{1}, a_{2}, d_{1}, d_{2}$.

Proposition 8.5. For any $m \in \mathbf{N},|\alpha| \leq m$ it holds

$$
\sum_{|\alpha| \leq m} \sup \left|D_{P_{j}}^{\alpha}\left[\left(A_{k}^{j}\left(P_{j}\right)\left(u_{1}\right)_{0}^{\sim}\right)\left(P_{j}\right)\right]\right| \leq c_{k}^{j}\left\|u_{1}\right\|_{L^{1, m}\left(\mathbb{R}_{+}^{3} ; X\right)}
$$

where

$$
c_{k}^{j}(m)=\text { const. }\left(\sum_{(l, j)} \sum_{|\alpha| \leq m} \sup \left|D_{P_{j}}^{\alpha} A_{k}^{j}\left(P_{j}\right)\right|\right)\left(\sum_{(l, j)} \sum_{|\alpha| \leq m} \sup \left|D_{P_{j}}^{\alpha} B_{k}^{j}\left(P_{j}\right)\right|\right)
$$

and const. is a number depending only on the coefficients $a_{1}, a_{2}, d_{1}, d_{2}$.

\section{REFERENCES}

[1] A. Adams, Sobolev spaces, Academic Press, New York, London, 1975.

[2] H.-D. Alber and R. Leis, Initial boundary value and scattering problems in mathematical physics, in: Partial differential equations and calculus of variations, Springer, Berlin, 1988, 23-60.

[3] F. Ali Mehmeti, Regular solutions of transmission and interaction problems for wave equations, Math. Methods Appl. Sci. 11 (1989), 665-685.

[4] F. Ali Mehmeti, Spectral theory and $L^{\infty}$-time decay estimates for Klein-Gordon equations on two half axes with transmission: the tunnel effect, Math. Methods Appl. Sci. 17 (1994), 697-752.

[5] F. Ali Mehmeti, R. Haller-Dintelmann and V. Regnier, The influence of the tunnel effect on $L^{\infty}$-time decay, in: Spectral theory, mathematical system theory, evolution equations, differential and difference equations. Birkhäuser, Basel, 2012, 11-24.

[6] F. Ali Mehmeti, E. Meister and K. Mihalinčić, Spectral theory for the wave equation in two adjacent wedges, Math. Methods Appl. Sci. 20 (1997), 1015-1044.

[7] L. Aloui, S. Ibrahim and K. Nakanishi, Exponential energy decay for damped KleinGordon equation with nonlinearities of arbitrary growth, Comm. Partial Differential Equations 36 (2011), 797-818.

[8] D. Andrade, L.H. Fatori and J.E. Muñoz Rivera, Nonlinear transmission problem with a dissipative boundary condition of memory type, Electron. J. Differential Equations 2006, No. 53, 16 pp.

[9] M. Beals and W. Strauss, $L^{p}$-estimates for the wave equation with a potential, Comm. Partial Differential Equations 18 (1993), 1365-1397.

[10] M. Daoulatli, Energy decay rates for solutions of the wave equation with linear damping in exterior domain, Evol. Equ. Control Theory 5 (2016), 37-59.

[11] M. Daoulatli, Energy decay rates for solutions of the wave equations with nonlinear damping in exterior domain, J. Differential Equations 264 (2017), 4260-4302.

[12] W.R. Green, Time decay estimates for the wave equation with potential in dimension two, J. Differential Equations 257 (2014), 868-919.

[13] L. Hörmander, The analysis of linear partial differential operators. I., Springer-Verlag, Berlin, 1983.

[14] J.B. Jeong, Nonlinear transmission problem for wave equation with boundary condition of memory type, Acta Appl. Math. 110 (2010), 907-919.

[15] F. John, Existence for large times of strict solutions of nonlinear wave equations in three space dimensions for small initial data, Comm. Pure Appl. Math. 40 (1987), 79-109. 
[16] F. John and S. Klainerman, Almost global existence to nonlinear wave equations in three space dimensions, Comm. Pure Appl. Math. 37 (1984), 443-455.

[17] S. Klainerman and G. Ponce, Global, small amplitude solutions to nonlinear evolution equations, Comm. Pure Appl. Math. 36 (1983), 133-141.

[18] R. Leis, Initial boundary value problems in mathematical physics, B.G. Teubner, Stuttgart, J. Wiley, Chichester, 1986.

[19] O. Liess, Decay estimates for the solutions of the system of crystal optics, Asymptotic Anal. 4 (1991), 61-95.

[20] B. Marshall, W. Strauss and S. Wainger, $L^{p}-L^{q}$ estimates for the Klein-Gordon equation, J. Math. Pures Appl. 59 (1980), 417-440.

[21] E. Meister, Some solved and unsolved canonical transmission problems of diffraction theory, in Problems and methods in mathematical physics, Teubner, Stuttgart, 1994., 89-99.

[22] K. Mihalinčić, Time decay estimates for the wave equation with transmission and boundary conditions, Dissertation, Technische Universität Darmstadt, Germany, 1998.

[23] K. Mihalinčić, Wave equation in halfspace and in two adjacent wedges, ZAMM Z. Angew. Math. Mech. 78 (1998) S3, 1017-1018.

[24] R. Racke, Lectures on nonlinear evolution equations, Friedr. Vieweg \& Sohn, Braunschweig, 1992.

[25] M. Reed and B. Simon, Methods of modern mathematical physics. I. Functional analysis, Academic Press, New York and London, 1972.

[26] W. von Wahl, $L^{p}$ - decay rates for homogeneous wave-equations, Math. Z. 120 (1971), 93-106.

[27] P. Werner, Resonance phenomena in infinite strings with periodic ends, Math. Methods Appl. Sci. 17 (1994), 115-154.

[28] C.H. Wilcox, Sound propagation in stratified fluids, Springer-Verlag, New York, 1984.

[29] E.C. Zachmanoglou, The decay of solutions of the initial-boundary value problem for the wave equation in unbounded regions, Arch. Rational Mech. Anal. 14 (1963), 312325.

[30] A.H. Zemanian, Distribution theory and transform analysis. An introduction to generalized functions, with applications, McGraw-Hill, New York-Toronto-London-Sydney, 1965.

K. Mihalinčić

Faculty of Tourism and Hospitality Management

University of Rijeka

Primorska 42, 51410 Opatija

Croatia

E-mail: kresom@fthm.hr

Received: 29.5.2017.

Revised: 18.5.2018. 